\title{
Optimal consensus set for digital line and plane fitting
}

\author{
Rita Zrour, ${ }^{1}$ Yukiko Kenmochi, ${ }^{2}$ Hugues Talbot, ${ }^{2}$ Lilian Buzer, ${ }^{2}$ Yskandar Hamam, ${ }^{2,3}$ Ikuko Shimizu, ${ }^{4}$ Akihiro Sugimoto ${ }^{5}$ \\ 1'Laboratory XLIM, SIC Department, University of Poitiers, UMR CNRS 6712, France \\ ${ }^{2}$ Université Paris-Est, Laboratoire d'Informatique Gaspard-Monge, ESIEE-CNRS, France
}

${ }^{3}$ F'SATI - Tshwane University of Technology, Pretoria, South Africa

${ }^{4}$ Tokyo University of Agricult ure and Technology, Japan

${ }^{5}$ National Instit ute of Informatics, Japan

ABSTRACT: This paper presents a new method for fitting a digital line or plane to a given set of points in a $2 \mathrm{D}$ or $3 \mathrm{D}$ image in the presence of noise by maximizing the number of inliers, namely the consensus set. By using a digital model instead of a continuous one, we show that we can generate all possible consensus sets for model fitting. We present a deterministic algorithm that efficiently searches the optimal solution with time complexity $O\left(N^{d} \log N\right)$ for dimension $d$, where $d=2,3$, together with space complexity $O(N)$ where $N$ is the number of points.

Key words: line fitting; plane fitting; digital geometry; discrete optimization; consensus set

\section{Introduction}

Line fitting and plane fitting are essential tasks in the field of image analysis and computer vision. For instance, these procedures are useful for shape approximation (Bhowmick and Bhaattacharya, 2007; Sivignon et al., 2004), image registration (Zitova and Flusser, 2003; Shum et al., 1995), and image segmentation (Köster and S $\square$ pann, 2000; Kenmochi et al., 200 $\square 8$ and considered as the problem of parameter estimation (Hartley and Zisserman, 2003). There exist several optimal methods for fitting such as least-s quare fitting, least-absolute-value fitting or least median of s quares (LMS) (Boyd and Vandenberghe, 2004; Press et al., 2007). In these methods, a continuous line or plane model is used, defined respectively by

$$
\begin{aligned}
& \boldsymbol{L}=\left\{(x, y) \in R^{2}: a x+y+b=0\right\} \\
& \boldsymbol{P}=\left\{(x, y, z) \in R^{3}: a x+b y+c+z=0\right\}
\end{aligned}
$$

where $a, b, c \in R$. The fitting is carried out through optimizing different cost functions. For instance, least$\mathrm{s}$ quares minimizes the sum of the geometric distances from all given points to the model. The solution can be obtained analytically, however it is not robust to the presence of outliers, namely points which do not fit the model. Least-absolute values uses the vertical distances, instead of the geometric distances, for its minimization. Some efficient iterative algorithms have been proposed in the literature. However, if there are outliers, the solution is known to be unstable. In contrast, Least Median of S quares (LMS) minimizes the median of the vertical/geometric distances of all given points to the model. Thus, the fitting is robust as long as fewer than half of the given points are outliers (Rousseeuw, 19 $\square 84$ )

In this paper, we present a novel globally optimal method that, given an arbitrary cloud of $2 \mathrm{D}$ or $3 \mathrm{D}$ points, finds the line or plane that minimizes the number of outliers, or alternatively maximizing the number of inliers, namely points which do fit the model, also called the consensus set. The idea of using such consensus sets was proposed for the RANdom 
Sample Consensus (RANSAC) method (Fischler and Bolles, 1981), which is one of the most widely used in the field of computer vision. However RANSAC (and its variations) is inherently probabilistic in its approach, and do not guarantee any optimality while our method is both deterministic and optimal in the size of the consensus set. In order to guarantee the optimality of consensus sets, we follow a digital geometry methodology (Klette and Rosenfeld, 2004) by using a digital line and plane models (Klette and Rosenfeld, 2004; Reveilles, 1991) instead of (1) and (2). This methodology is in fact natural given the assumption that our inputs are digital images. Besides, such a digital model allows us to distinguish between digitization-induced noise and actual noise. Related work using digital line or plane models can be found in works such as digital line or plane recognition (Buzer, 2003; Buzer, 2006; Gerard et al., 2005), digital curve polygonalisation (Bhowmick and Bhaattacharya, 2007; Debled Rennesson et al., 2006), and digital surface polyhedrization (Provot et al., 2006; Sivignon et al., 2004) with and without the presence of noise. However, to the best of our knowledge, outliers, namely points which do not fit the model, have never been studied in the field of digital geometry.

This work is an extension of our previous papers on digital line fitting (Zrour et al., 2009) and digital plane fitting (Zrour et al., 2009). We can treat both the 2D and $3 \mathrm{D}$ problems by considering them in the dual space of the dual transform (De Berg, 2008). Indeed, we show that digital plane fitting in 3D can be treated with a similar methodology to the one for digital line fitting in $2 \mathrm{D}$ because it can be viewed as a $2 \mathrm{D}$ problem. We present an algorithm that has a time complexity of $O\left(N^{d} \log N\right)$ for the dimension $d=2,3$, with $N$ the number of points, and a space complexity $O(N)$. Note that the space complexity is not affected by the dimension. We also point out that there are degenerate cases since our inputs are only integers and all computations can be executed by using rational numbers alone, and present how we can deal with them.

The rest of the paper is as follows: in section II we expose the framework of our digital model. In section III we prove the optimality of our result. In sections IV and $\mathrm{V}$, we provide an algorithm for the computation of the line fit in 2D and an extended algorithm for the plane fit in 3D, respectively. Section VI provides a method for extracting the parameters from the fit. Sections VII is devoted to $2 \mathrm{D}$ and $3 \mathrm{D}$ results and applications respectively. Finally Section VIII states some conclusions and perspectives.

\section{The problem of digital line and plane fitting}

A line $\boldsymbol{L}$ and a plane $\boldsymbol{P}$ in the Euclidean space $R^{n}$, $n=2,3$, are defined by (1) and (2). In this paper, we use digital models, instead of continuous ones, for lines and planes in a discrete space $Z^{d}$ for $d=2,3$, where $Z$ is the set of all integers. We contend that using digital models is natural when our input data is a set of points in a digital space.

A digital line $\boldsymbol{D}(\boldsymbol{L})$ that is the digitization of $\boldsymbol{L}$ is defined by the set of discrete points satisfying two inequalities:

$$
\boldsymbol{D}(\boldsymbol{L})=\left\{(x, y) \in Z^{2}: 0 \leq a x+y+b \leq w\right\}
$$

where $w$ is a given constant value. Geometrically, $\boldsymbol{D}(\boldsymbol{L})$ is a set of discrete points lying between two parallel lines $a x+y+b=0$ and $a x+y+b=w$, and $w$ specifies the vertical distance between them. From the digital geometrical viewpoint (Klette and Rosenfeld, 2004; Reveilles, 1991), $w$ should not be less than 1 if we expect that $\boldsymbol{D}(\boldsymbol{L})$ to be 8-connected. In other words, 1 is the minimum distance to keep the connectivity of a digital line. We can also fix the horizontal distance, instead of the vertical one, between the two parallel lines. In that case, we simply exchange $x$ and $y$ in (3).

A digital plane $\boldsymbol{D}(\boldsymbol{P})$ that is the digitization of $\boldsymbol{P}$ is defined by

$$
\boldsymbol{D}(\boldsymbol{P})=\left\{(x, y, z) \in Z^{3}: 0 \leq a x+b y+z+c \leq\right.
$$

$w\}(4)$

and similar discussions on the value setting for $w$ and its direction choice among the $x-, y-$ and $z$-axis directions, namely, the permutation of $x, y$ and $z$ in (4), are also valid for $\boldsymbol{D}(\boldsymbol{P})$.

Using the above digital line and plane models, our fitting problem is then described as follows: given a finite set of discrete points such that

$$
\boldsymbol{S}=\left\{\boldsymbol{x}_{\boldsymbol{i}} \in Z^{d}: i=1,2, \cdots, N\right\},
$$

we would like to find a digital line $\boldsymbol{D}(\boldsymbol{L})$ for $d=\mathbf{2}$ (resp. a digital plane $\boldsymbol{D}(\boldsymbol{P})$ for $d=3$ ) such that $\boldsymbol{D}(\boldsymbol{L})$ 
(resp. $\boldsymbol{D}(\boldsymbol{P})$ ) contains the maximum number of points in $\boldsymbol{S}$. Points $\boldsymbol{x}_{\boldsymbol{i}} \in \boldsymbol{S}$ are called inliers if $\boldsymbol{x}_{\boldsymbol{i}} \in \boldsymbol{S} \cap \boldsymbol{D}(\boldsymbol{L})$ (resp. $\boldsymbol{x}_{\boldsymbol{i}} \in \boldsymbol{S} \cap \boldsymbol{D}(\boldsymbol{P})$ ); otherwise, they are called outliers.

\section{Digital models and their consensus sets}

Our approach is focusing on inlier sets, also called consensus sets. Since the size of $\boldsymbol{S}$ is finite and each element $\boldsymbol{x} \in \boldsymbol{S}$ has finite coordinates, we easily notice that the number of different consensus sets for the digital line or plane fitting of $\boldsymbol{S}$ is finite as well. Thus, if we can find all different consensus sets $\boldsymbol{C}$ from a given $\boldsymbol{S}$, we just need to verify the size of each $\boldsymbol{C}$ and find the maximum one (ones if there are several) as the optimal solution.

Then the following question comes up naturally: is it possible to find all the consensus sets of $\boldsymbol{S}$ ? If the answer is positive, how can we do it? In this section, we will answer these questions. For the followings, we give some notions related to digital lines and planes. Two parallel lines (resp. planes) that are given by the equations in (3) (resp. (4)) are called the support lines (resp. planes) of a digital line (resp. plane). Discrete points that are on support lines (resp. planes) are called critical points of a digital line (resp. plane).

\section{A. Digital lines and their consensus sets}

We first attack the 2D case of digital line fitting.

Proposition 1 Let $\boldsymbol{C}$ be a consensus set of $\boldsymbol{S}$ for a digital line. It is possible to find a new digital line whose consensus set is the same as $\boldsymbol{C}$ such that it has at least two critical points.

Proof Let $\boldsymbol{D}$ be an initial digital line that contains all points of $\boldsymbol{C}$ as its inliers. Then, the following three cases can be considered when studying the critical points of $\boldsymbol{D}$.

1. Suppose that $\boldsymbol{D}$ has more than one critical points, then the proposition is already established in this case.

2. Suppose that $\boldsymbol{D}$ has one critical point $\boldsymbol{p}_{\mathbf{1}}$. In this case, we apply a rotation to $\mathbf{D}$ around $\boldsymbol{p}_{\mathbf{1}}$ until finding another point $\boldsymbol{p}_{\mathbf{2}}$ in $\boldsymbol{C}$ so that $\boldsymbol{p}_{\mathbf{2}}$ becomes a critical point. The rotation is accomplished in such a way as to maintain the distance $w$ between the support lines, and so that the support line on which there is not $\boldsymbol{p}_{\mathbf{1}}$ is rotated around the point $\boldsymbol{p}_{\mathbf{1}}^{\prime}$ that is the projection of $\boldsymbol{p}_{\mathbf{1}}$ on the line. Figure 1 shows an example of a rotated digital line. Note that we can rotate $\boldsymbol{D}$ either clockwise or counterclockwise.

3. Suppose that $\boldsymbol{D}$ has no critical point. In this case, we first apply a translation to $\boldsymbol{D}$ in order to find a first critical point $\boldsymbol{p}_{\mathbf{1}}$. Note that a translation can be made to any direction and the two support lines shall maintain the distance $\boldsymbol{w}$ between them. During such a translation, if more than one points are detected as critical points, then the proof is complete. If just one point $\boldsymbol{p}_{\mathbf{1}}$ is detected, as illustrated in Figure 2, then a rotation is made around $\boldsymbol{p}_{\mathbf{1}}$ as mentioned in the previous case, in order to obtain a second critical point $\boldsymbol{p}_{2}$.

From this proposition, we see that we can find a digital line $\boldsymbol{D}(\boldsymbol{L})$ for any consensus set $\boldsymbol{C}$ of $\boldsymbol{S}$ such that it has at least two critical points. This is intuitively understandable, because when we move a digital line $\boldsymbol{D}(\boldsymbol{L})$ in the image plane, its consensus set $\boldsymbol{C}$ will change when a critical point goes out from $\boldsymbol{D}(\boldsymbol{L})$, namely, becomes an outlier, due to the motion of the line. Indeed, such a digital line $\boldsymbol{D}(\boldsymbol{L})$ can be constructed from a pair of points chosen from $\boldsymbol{S}$ such that they become critical points of $\boldsymbol{D}(\boldsymbol{L})$. Consequently, we can find all $\boldsymbol{C}$ from those $\boldsymbol{D}(\boldsymbol{L})$ constructed from pairs of points in $\boldsymbol{S}$.

\section{B. Digital planes and their consensus sets} Similarly to digital lines, we have the following proposition for digital planes.

Proposition 2 Let $\boldsymbol{C}$ be a consensus set of $\boldsymbol{S}$ for a digital plane. It is possible to find a new digital plane whose consensus set is the same as $\boldsymbol{C}$ such that it has at least three critical points.

Proof Let $\boldsymbol{D}$ be an initial digital plane that contains all points in $\boldsymbol{C}$ as its inliers. Then, the following four cases can be considered when observing the critical points of D.

1. Suppose that $\boldsymbol{D}$ has more than two critical points, then the proposition is correct in this case. 
2. Suppose that $\boldsymbol{D}$ has two critical points $\boldsymbol{p}_{1}$ and $\boldsymbol{p}_{2}$, which may be located on one side or either side of the two parallel support planes of $\boldsymbol{D}$. First, we take the projections $\boldsymbol{p}_{1}^{\prime}$ (resp. $\boldsymbol{p}_{2}^{\prime}$ ) of $\boldsymbol{p}_{1}$ (resp. $\boldsymbol{p}_{2}$ ) on the other support plane where $\boldsymbol{p}_{1}$ (resp. $\boldsymbol{p}_{2}$ ) does not exist in the $z$-axis direction. We then apply a rotation to $\boldsymbol{D}$ in such a way as to maintain the distance $w$ between the support planes until finding another point $\boldsymbol{p}_{3}$ in $\boldsymbol{C}$ so that $\boldsymbol{p}_{3}$ becomes a critical point. To achieve this to the support plane where $\boldsymbol{p}_{1}$ and $\boldsymbol{p}_{\mathbf{2}}$ exist, we apply a rotation around the line going through $\boldsymbol{p}_{\mathbf{1}}$ and $\boldsymbol{p}_{\mathbf{2}}$, as illustrated in Figure 3. To the other support plane, we apply a rotation around the line going through $\boldsymbol{p}_{\mathbf{1}}^{\prime}$ and $\boldsymbol{p}_{2}^{\prime}$, as illustrated in Figures 3 . In the case of Figure 4, similarly, to the support plane where $\boldsymbol{p}_{1}$ (resp. $\boldsymbol{p}_{2}$ ) exists, we apply a rotation around the line going through $\boldsymbol{p}_{\mathbf{1}}$ and $\boldsymbol{p}_{\mathbf{2}}^{\prime}$ (resp. $\boldsymbol{p}_{\mathbf{1}}^{\prime}$ and $\boldsymbol{p}_{2}$ ). Note that we can rotate $\boldsymbol{D}$ either clockwise or counterclockwise.

3. Suppose that $\boldsymbol{D}$ has one critical point $\boldsymbol{p}_{\mathbf{1}}$. In this case, we also consider the projection of $\boldsymbol{p}_{1}, \boldsymbol{p}_{1}^{\prime}$. We then apply a rotation to each support plane until finding another point $\boldsymbol{p}_{\mathbf{2}}$ in $\boldsymbol{C}$ so that $\boldsymbol{p}_{\mathbf{2}}$ becomes a critical point, as illustrated in Figure 5. The support plane where $\boldsymbol{p}_{\mathbf{1}}$ (resp. $\boldsymbol{p}_{\mathbf{1}}^{\prime}$ ) exists is rotated around any line going through $\boldsymbol{p}_{1}$ (resp. $\boldsymbol{p}_{\mathbf{1}}^{\prime}$ ) on the support plane. If just one point $\boldsymbol{p}_{\mathbf{2}}$ is found as a second critical point after the rotation, then another rotation is made, as mentioned in the previous case, in order to obtain a third critical point $\boldsymbol{p}_{\mathbf{3}}$.

4. Suppose that $\boldsymbol{D}$ has no critical point. In this case, we first apply a translation to $\boldsymbol{D}$ in order to find a first critical point $\boldsymbol{p}_{\mathbf{1}}$. Note that a translation can be made in any direction while the two support planes maintain a constant distance $w$ between them. During such a translation, if more than two points are found as critical points, then the proof is complete. If just one point $\boldsymbol{p}_{1}$ is found, as illustrated in Figure 6, then we follow the previous case.

From this proposition, similarly to the $2 \mathrm{D}$ case, we see that we can find a digital plane $\boldsymbol{D}(\boldsymbol{P})$ for any consensus set $\boldsymbol{C}$ of $\boldsymbol{S}$ such that it has at least three critical points. Consequently, we can find all $\boldsymbol{C}$ from $\boldsymbol{D}(\boldsymbol{P})$ constructed from all possible triplets of points in $\boldsymbol{S}$.

\section{Digital line fitting algorithm}

We first describe the digital line fitting problem in the dual space of the duality transform (De Berg, 2008), because our algorithm works in the dual space. We then present an algorithm to exhibit the optimal consensus set (or sets if the solution is not unique) that maximizes the number of inliers of a fitted digital line from a given set $\boldsymbol{S}$ of $2 \mathrm{D}$ discrete points, step by step. We also describe special treatments for degenerate cases; it should be noted that digital images likely present many degenerate cases that must be processed separately.

\section{A. Digital line fitting in the dual space}

Our algorithm is inspired by the algorithm of LMS (Souvaine and Steele, 1987) working in the dual space of the following duality transform (De Berg, 2008): let $\boldsymbol{p}=\left(x_{\boldsymbol{p}}, y_{\boldsymbol{p}}\right)$ be a 2D point in the primal space $(x, y)$ then the dual of $\boldsymbol{p}$ is the line:

$$
L_{\boldsymbol{p}}^{0}=\left\{(a, b): x_{\boldsymbol{p}} a+b+y_{\boldsymbol{p}}=0\right\}
$$

in the dual space $(a, b)$. Likewise, the dual of a nonvertical line $a x+y+b=0$ in the primal space is the point $(a, b)$ in the dual space.

Now, let us consider the dual-space interpretation of a digital line in the primal space, defined by (3). A digital line is regarded as a set of two parallel lines whose slopes are $-a$, and whose $y$-intercepts are at $-b$ and $w-b$. It corresponds, in the dual space, to a vertical line segment of length $w$ which is the distance between two parallel lines of the digital line, as illustrated in Figure 7. Because points in $\boldsymbol{S}$ in the primal space are represented by lines in the dual space, the problem of finding the optimal consensus set in the primal set is equivalent to searching the best position of the vertical line segment of length $w$ such that it intersects with as many lines as possible in the dual space, as illustrated in Figure 7.

\section{B. Strips made from a critical point}

Obviously, we cannot search everywhere in the dual space to find the best line segment. From Proposition 1, we know that, for any consensus set, there exists a digital line that features at least two critical points. 
Therefore, we first take one point $\boldsymbol{p} \in \boldsymbol{S}$, and consider it to be the first critical point of such a fitted digital line. Because $\boldsymbol{p}$ corresponds to a line $L_{\boldsymbol{p}}^{0}$ in the dual space, all digital lines for which $\boldsymbol{p}$ is a critical point correspond to the set of all the vertical line segments of length $w$ having one of its endpoints on $L_{\boldsymbol{p}}^{0}$ in the dual space, as shown in Figure 8. The set of such digital lines, therefore, forms two strips in the dual space; one of them is bounded by $L_{\boldsymbol{p}}^{0}$ and $L_{\boldsymbol{p}}^{1}$, and another is bounded by $L_{\boldsymbol{p}}^{0}$ and $L_{\boldsymbol{p}}^{2}$, where

$$
\begin{aligned}
& L_{\boldsymbol{p}}^{1}=\left\{(a, b): x_{\boldsymbol{p}} a+b+y_{\boldsymbol{p}}+w=0\right\}, \\
& L_{\boldsymbol{p}}^{2}=\left\{(a, b): x_{\boldsymbol{p}} a+b+y_{\boldsymbol{p}}-w=0\right\},
\end{aligned}
$$

as illustrated in Figure 8. For simplification, we focus on the strip bounded by $L_{\boldsymbol{p}}^{0}$ and $L_{\boldsymbol{p}}^{1}$, because the following discussion is also valid for another strip bounded by $L_{\boldsymbol{p}}^{0}$ and $L_{\boldsymbol{p}}^{2}$.

\section{Digital lines with critical point pairs}

According to Proposition 1, we choose a point $\boldsymbol{q} \in \boldsymbol{S} \backslash\{\boldsymbol{p}\}$ to be the second critical point of a fitted digital line such that $x_{q} \neq x_{p}$; the case of $x_{q}=x_{p}$ will be discussed later. Any point $\boldsymbol{q}$ in the primal space is represented by the line $L_{\boldsymbol{q}}^{0}$ in the dual space, as shown in Figure 9. We see in this figure that $L_{\boldsymbol{q}}^{0}$ intersects each of the strip boundaries, $L_{p}^{0}$ and $L_{\boldsymbol{p}}^{1}$, if it is not parallel to $L_{p}^{0}$; the parallel case occurs when $x_{\boldsymbol{q}}=x_{\boldsymbol{p}}$, and it will be dealt with separately as a degenerate case in Section F. The intersections between $L_{\boldsymbol{q}}^{0}$ and $L_{\boldsymbol{p}}^{i}$, $\boldsymbol{\sigma}_{\boldsymbol{q}}^{i}=\left(a_{\boldsymbol{q}}^{i}, b_{\boldsymbol{q}}^{i}\right)$, for $i=0,1$, are calculated. Geometrically, the vertical line segment in the strip, one of whose endpoints is one of the intersections $\boldsymbol{\sigma}_{\boldsymbol{q}}^{i}$, in the dual space corresponds to a digital line with the two critical points $\boldsymbol{p}$ and $\boldsymbol{q}$ in the primal space. This shows that the digital lines corresponding to the vertical line segments between the two intersections $\boldsymbol{\sigma}_{\boldsymbol{q}}^{0}$ and $\boldsymbol{\sigma}_{\boldsymbol{q}}^{1}$ in the strip always contain $\boldsymbol{q}$ as an inlier.

\section{Finding the largest consensus set in a strip}

In order to know the number of inliers within the digital lines with a critical point $\boldsymbol{p}$, we check the intersections $\boldsymbol{\sigma}_{\boldsymbol{q}}^{0}$ and $\boldsymbol{\sigma}_{\boldsymbol{q}}^{1}$ of $L_{\boldsymbol{q}}^{0}$ for all $\boldsymbol{q} \in \boldsymbol{S} \backslash\{\boldsymbol{p}\}$ with the strip boundaries, $L_{\boldsymbol{p}}^{0}$ and $L_{\boldsymbol{p}}^{1}$. We use two values $f_{\boldsymbol{q}}^{i}$ for $i=0,1$, which is set to be 1 if $L_{\boldsymbol{q}}^{0}$ enters the strip from $L_{\boldsymbol{p}}^{i}$, and -1 if $L_{\boldsymbol{q}}^{0}$ leaves the strip from $L_{\boldsymbol{p}}^{i}$.

Once the intersections $\boldsymbol{\sigma}_{\boldsymbol{q}}^{i}=\left(a_{\boldsymbol{q}}^{i}, b_{\boldsymbol{q}}^{i}\right)$, and the associated value $f_{\boldsymbol{q}}^{i}$ for $i=0,1$ are calculated for all $\boldsymbol{q} \in \boldsymbol{S} \backslash\{\boldsymbol{p}\}$, we sort all the triples $\left(a_{\boldsymbol{q}}^{i}, b_{\boldsymbol{q}}^{i}, f_{\boldsymbol{q}}^{i}\right)$ in increasing order by using $a_{\boldsymbol{q}}^{i}$ as keys. As for determining the location of the maximum number of inliers, a function $F(a)$ is used; after initially setting $F(a)=1$ for every $a$, since we already know that $\boldsymbol{p}$ is an inlier, then the value $f_{\boldsymbol{q}}^{i}$ is added to $F(a)$ for $a \geq a_{\boldsymbol{q}}^{i}$ in the above sorted order. By looking for the maximum value of $F(a)$, we obtain the parameter set $(a, b)$ corresponding to the maximum optimal consensus set for a critical points $\boldsymbol{p}$. In this section, we consider that all $L_{\boldsymbol{q}}^{0}$, enter or leave a strip at different $a$. The degenerate cases such that many lines $L_{\boldsymbol{q}}^{0}$ enter or leave a strip at the same $a$ will be described in Section F.

\section{E. Algorithm}

We now present Algorithm 1 in Figure 10. Input is a set $\boldsymbol{S}$ of discrete points and a distance value $w$ of our digital line model. Output is a set $\boldsymbol{V}$ of parameter values $\left(a^{C}, b^{C}\right)$ corresponding to the fitted digital lines of that give the optimal consensus sets. In the algorithm, we consider another strip bounded by $L_{\boldsymbol{p}}^{0}$ and $L_{\boldsymbol{p}}^{2}$ as well, as seen in Steps 4, 9 and 21. We remark that, because $b_{\boldsymbol{q}}^{i}$ is not used for the sorting step and can be calculated from $a_{\boldsymbol{q}}^{i}$, we do not have to store it for each intersection. Simply for a candidate of the optimal consensus set, we calculate it as shown in Steps 21 and 22. Note that, depending on the strip, we calculate different $b^{C}$ because of the translation difference $w$ between the two strips. We also remark that Algorithm 1 provides us with the set of parameter pair values $\left(a^{C}, b^{C}\right)$ of all the fitted digital lines of (3) that give the optimal consensus sets.

The time complexity of the algorithm is $O\left(N^{2} \log N\right)$, because we have $N$ points in $\boldsymbol{S}$ and each $\boldsymbol{p} \in \boldsymbol{S}$ needs the complexity $O(N \log N)$, for sorting at most $2 N-2$ different values $a_{\boldsymbol{q}}^{i}$ for $\boldsymbol{q} \in \boldsymbol{S}, \boldsymbol{q} \neq \boldsymbol{p}$, and $i=0,1$. The 
space complexity is $O(N)$ because for each sorting we have at most $2 N-2$ different pairs $\left(a_{k}, f_{k}\right)$.

Because all inputs can be given as integers or rational numbers, all computations in Algorithm 1 can be made by using only rational numbers. This guarantees that all results obtained by Algorithm 1 contain no numerical error. However, degenerate cases may occur, which are discussed in the followings.

\section{F. Degenerate cases}

In this section, we deal with degenerate cases, which are not considered in Algorithm 1. They are summarized as follows:

- Suppose that $\boldsymbol{p}$ and $\boldsymbol{q}$ such that $x_{\boldsymbol{q}}=x_{\boldsymbol{p}} ; L_{\boldsymbol{q}}^{0}$ is parallel to $L_{\boldsymbol{p}}^{0}$. If ; $L_{\boldsymbol{q}}^{0}$ is between $L_{\boldsymbol{p}}^{0}$ and $L_{\boldsymbol{p}}^{1}$ (resp. $L_{\boldsymbol{p}}^{2}$ ), then we set the initial value of the function $F(a)$ to 2 when $l=1$ (resp. $l=2$ ) because $\boldsymbol{q}$ is an inlier for any $a$. Otherwise, we set it to 1, as described in Algorithm 1, because $\boldsymbol{q}$ is an outlier for any $a$.

- When many lines $L_{\boldsymbol{q}}$ enter or leave a strip at the same moment $a$, all the positive values $f_{q}^{i}$ of that moment must be added to the function $F(a)$ at once (Step 17 in Algorithm 1), and the value $F(a)$ is compared with the current maximum value Max (Step 18 in Algorithm 1). Note that all the negative valued $f_{\boldsymbol{q}}^{i}$ of the same moment $a$ must be added after the comparison to the function $F(a)$. Indeed such a point $\boldsymbol{q}$ must be considered as an inlier until that moment.

Obviously, those modifications affect neither the time nor space complexity of the algorithm.

\section{Digital plane fitting algorithm}

The algorithm is based on a similar idea to the one for 2D digital line fitting, presented in the previous section. The key idea for the extension to 3D digital plane fitting is treating the $3 \mathrm{D}$ problem as a $2 \mathrm{D}$ problem. In this section, we show how to reduce the dimension from three to two, and obtain an algorithm providing a $O\left(N^{3} \log N\right)$ time and $O(N)$ space complexity.

\section{A. Digital plane fitting in the dual space}

A point $\boldsymbol{p}=(x, y, z)$ in the primal space associates to a non-vertical plane

$$
P_{\boldsymbol{p}}=\{(a, b, c): x a+y b+c+z=0\}
$$

in the dual space. Conversely, a non-vertical plane in the primal space associates to a point in the dual space. Similarly to a digital line, a digital plane defined by (4) is regarded as a set of non-vertical parallel planes whose normal vectors are $(a, b, 1)$ and whose $z$ intercepts are between $-c$ and $w-c$, and it forms a vertical line segment of length $w$ in the dual space as illustrated in Figure 11. The problem of finding the optimal consensus set for digital plane fitting in the primal space is then equivalent to searching the position of the vertical line segment of length $w$ such that it intersects with the maximum number of planes in the dual space.

We now need a search procedure for an optimal segment. Thanks to Proposition 2, we know that, for any consensus set, there exists a digital plane featuring at least three critical points, among which at least two are on one of the support planes. Thus, taking two different points $\boldsymbol{p}, \boldsymbol{q}$ from $\boldsymbol{S}$ in the primal space, we first consider all the digital planes on which both $\boldsymbol{p}$ and $\boldsymbol{q}$ are critical points on the same support plane. In the dual space, digital planes having two critical points $\boldsymbol{p}$, $\boldsymbol{q}$ forms two strips, which will be described in Section B. We then explain how digital planes with two critical points $\boldsymbol{p}, \boldsymbol{q}$ appear in the strips when they have a third critical point $\boldsymbol{r}$, so that the sub-problem becomes the same as the 2D sub-problem.

\section{B. Strips made from a critical point pair}

Let $\boldsymbol{p}=\left(x_{\boldsymbol{p}}, y_{\boldsymbol{p}}, z_{\boldsymbol{p}}\right)$ and $\boldsymbol{q}=\left(x_{\boldsymbol{q}}, y_{\boldsymbol{q}}, z_{\boldsymbol{q}}\right)$. In the dual space, they represent two planes $P_{\boldsymbol{p}}$ and $P_{\boldsymbol{q}}$, defined by (7). They intersect in a line $L_{\boldsymbol{p} \boldsymbol{q}}^{0}$ if $\boldsymbol{p}$ and $\boldsymbol{q}$ are chosen such that $\left(x_{p}-x_{q}\right)^{2}+\left(y_{p}-y_{q}\right)^{2} \neq 0$; otherwise, $P_{p}$ and $P_{\boldsymbol{q}}$ are parallel, and no intersection line can be found. The intersection line $L_{p q}^{0}$ is represented by the following equation:

$$
L_{\boldsymbol{p q}}^{0}=\{\boldsymbol{v}=(a, b, c): \boldsymbol{v}=\boldsymbol{u}+t \boldsymbol{d}, t \in R\},
$$
where

$$
\begin{gathered}
d=\left(x_{\boldsymbol{p}}, y_{\boldsymbol{p}}, 1\right) \wedge\left(x_{\boldsymbol{q}}, y_{\boldsymbol{q}}, 1\right)=\left(y_{\boldsymbol{p}}-y_{\boldsymbol{q}}, x_{\boldsymbol{q}}-\right. \\
\left.x_{\boldsymbol{p}}, x_{\boldsymbol{p}} y_{\boldsymbol{q}}-x_{\boldsymbol{q}} y_{\boldsymbol{p}}\right),
\end{gathered}
$$


and $\boldsymbol{u}=\left(u_{a}, u_{b}, u_{c}\right) ; \boldsymbol{u}$ is a chosen point on $L_{p \boldsymbol{q}}^{0}$. For example, if $x_{\boldsymbol{p}} y_{\boldsymbol{q}} \neq x_{\boldsymbol{q}} y_{\boldsymbol{p}}$, by fixing $u_{c}=0, u_{a}$ and $u_{b}$ are automatically found since $\boldsymbol{u}$ is on both $P_{\boldsymbol{p}}$ and $P_{\boldsymbol{q}}$.

Once $L_{\boldsymbol{p} \boldsymbol{q}}^{0}$ is found, then, all the digital planes on which both $\boldsymbol{p}$ and $\boldsymbol{q}$ are critical points on the same support plane in the primal space correspond to the set of all the vertical line segments of length $w$ having one of its endpoints on $L_{p q}^{0}$ in the dual space, as shown in Figure 12. We see in the figure that the set of such digital planes, therefore, forms two strips in the plane $Q_{p q}$ that contains $L_{\boldsymbol{p} \boldsymbol{q}}^{0}$ and the direction parallel to the $c$-axis. Taking the $d$-axis in $Q_{p q}$ as the orthogonal one to the $c$-axis, such $Q_{p q}$ is illustrated in Figures 12 and 13 . Each strip on $Q_{p q}$ illustrated in Figure 13 is bounded by two parallel lines, $L_{\boldsymbol{p} \boldsymbol{q}}^{0}$ and $L_{\boldsymbol{p} \boldsymbol{q}}^{i}$ for $i=1,2$, which are represented by:

$$
\begin{aligned}
& L_{\boldsymbol{p} \boldsymbol{q}}^{1}=\{\boldsymbol{v}=(a, b, c): \boldsymbol{v}=\boldsymbol{u}+\boldsymbol{e}+t \boldsymbol{d}, t \in R\}, \\
& L_{\boldsymbol{p} \boldsymbol{q}}^{2}=\{\boldsymbol{v}=(a, b, c): \boldsymbol{v}=\boldsymbol{u}-\boldsymbol{e}+t \boldsymbol{d}, t \in R\},
\end{aligned}
$$
where $e=(0,0, w)$. Note that they correspond to $L_{p}^{0}$ and $L_{\boldsymbol{p}}^{2}$ of (5) and (6) for the 2D case.

\section{Digital planes with critical point triplets}

Hereafter, we focus on one of the strips in $Q_{p q}$, because the following discussion is valid for both strips. Let us consider the strip bounded by $L_{p q}^{0}$ and $L_{p q}^{1}$, as illustrated in Figure 13. According to Proposition 2, we choose a point $\boldsymbol{r} \in \boldsymbol{S} \backslash\{\boldsymbol{p}, \boldsymbol{q}\}$ to be the third critical point of a fitted digital plane such that $\boldsymbol{r}$ is not colinear with $\boldsymbol{p}$ and $\boldsymbol{q}$; the colinear case will be handled separately as a degenerate case in Section F. Any point $\boldsymbol{r}$ in the primal space is represented by the line $L_{\boldsymbol{r}}$ in $Q_{p q}$ in the dual space, which is the intersection between $P_{r}$ and $Q_{p q}$, as shown in Figure 13. We see in this figure that $L_{r}$ intersects each of the strip boundaries, $L_{\boldsymbol{p} \boldsymbol{q}}^{0}$ and $L_{\boldsymbol{p} \boldsymbol{q}}^{1}$, if it is not parallel to $L_{\boldsymbol{p} \boldsymbol{q}}^{0}$; the parallel case will be also dealt with separately as a degenerate case in Section F. The intersections between $L_{\boldsymbol{r}}$ and $L_{\boldsymbol{p} \boldsymbol{q}}^{i}, \boldsymbol{\sigma}_{\boldsymbol{r}}^{i}=\left(a_{\boldsymbol{r}}^{i}, b_{\boldsymbol{r}}^{i}, c_{\boldsymbol{r}}^{i}\right)$, for $i=0,1$, are calculated from $L_{\boldsymbol{p} \boldsymbol{q}}^{i}$ and $P_{\boldsymbol{r}}$. Geometrically, the vertical line segment in the strip, one of whose endpoints is one of the intersections $\boldsymbol{\sigma}_{r}^{i}$, in the dual space corresponds to a digital plane with three critical points $\boldsymbol{p}, \boldsymbol{q}$ and $\boldsymbol{r}$ in the primal space. This indicates that the digital planes corresponding to the vertical line segments between the two intersections $\sigma_{r}^{0}$ and $\sigma_{r}^{1}$ in the strip always contain $\boldsymbol{r}$ as an inlier. This structure is already seen for the 2D case.

\section{Finding the largest consensus set in a strip}

Similarly to digital line fitting, in order to know the number of inliers within the digital planes with two critical points $\boldsymbol{p}$ and $\boldsymbol{q}$, we check the intersections $\boldsymbol{\sigma}_{\boldsymbol{r}}^{0}$ and $\boldsymbol{\sigma}_{r}^{1}$ of $L_{r}$ for all $\boldsymbol{r} \in \boldsymbol{S} \backslash\{\boldsymbol{p}, \boldsymbol{q}\}$ with the strip boundaries, $L_{\boldsymbol{p} \boldsymbol{q}}^{0}$ and $L_{\boldsymbol{p} \boldsymbol{q}}^{1}$. We use the similar function $f_{\boldsymbol{r}}^{i}$ for $\boldsymbol{r} \in \boldsymbol{S} \backslash\{\boldsymbol{p}, \boldsymbol{q}\}, \quad i=0,1$, and sort the quadruples $\left(a_{r}^{i}, b_{r}^{i}, c_{r}^{i}, f_{r}^{i}\right)$, instead of the triples for the 2D case, in increasing order by using either $a_{\boldsymbol{r}}^{i}$ or $b_{\boldsymbol{r}}^{i}$ as keys; if $Q_{\boldsymbol{p q}}$ is not perpendicular to the $a$-axis, we use $a_{r}^{i}$; otherwise, we use $b_{r}^{i}$. As for determining the location of the maximum number of inliers, we also use the similar function $F(a)$ (resp. $F(b)$ depending on the key selection) after initially setting $F(a)=2$ for every $a$. We obtain the parameter set $(a, b, c)$ corresponding to the maximum optimal consensus set for a pair of critical points $\boldsymbol{p}$ and $\boldsymbol{q}$. The degenerate cases such that many lines $L_{r}$ enter or leave a strip at the same intersection will be treated in the same manner as the 2D case.

\section{E. Algorithm}

We now present Algorithm 2 in Figure 14, which is easily obtained by modifying Algorithm 1. Input is a set $\boldsymbol{S}$ of discrete points and a distance value $w$ of our digital plane model. Output is a set $\boldsymbol{V}$ of parameter values $\left(a^{C}, b^{C}, c^{C}\right)$ corresponding to the fitted digital planes that represent the optimal consensus sets. In the algorithm, we consider another strip bounded by $L_{p q}^{0}$ and $L_{\boldsymbol{p} \boldsymbol{q}}^{2}$ in Steps 5, 10 and 22. We remark that, because $c_{r}^{i}$ is not used for the sorting step and can be calculated from $a_{\boldsymbol{r}}^{i}$ and $b_{\boldsymbol{r}}^{i}$, we do not have to store it for each intersection.

In Steps 11 and 15 , we only show the case where $a_{k}$ is used as keys for sorting. However, if $Q_{p q}$ is perpendicular to the $a$-axis, all $a_{k}$ has the same value. In such a case, as mentioned above, we use $b_{k}$ as keys, instead of $a_{k}$. The time complexity of the algorithm is 
$O\left(N^{3} \log N\right)$, because we have $N$ points in $S$ and each pair of $\boldsymbol{p}$ and $\boldsymbol{q}$ in $\boldsymbol{S}$ needs the complexity $O(N \log N)$ for sorting at most $2 N-4$ different values $a_{r}^{i}$ for $\boldsymbol{r} \in \boldsymbol{S} \backslash\{\boldsymbol{p}, \boldsymbol{q}\}$ and $i=0,1$. The space complexity is $O(N)$ because for each sorting we have at most $2 N-4$ different triples $\left(a_{k}, b_{k}, f_{k}\right)$.

All computations in Algorithm 2 can be also performed using only rational numbers since all inputs can be given as integers or rational numbers. This causes the following degenerate cases.

\section{F. Degenerate cases}

We consider the following three degenerate cases, where the second and third ones were already discussed for the 2D case in the previous section.

- If three points $\boldsymbol{p}, \boldsymbol{q}$ and $\boldsymbol{r}$ are colinear in the primal space, their associated planes $P_{\boldsymbol{p}}, P_{\boldsymbol{q}}$, and $P_{r}$ have a line intersection in the dual space. Therefore, for any digital plane having $\boldsymbol{p}$ and $\boldsymbol{q}$ as its critical points also has $\boldsymbol{r}$ as its another critical point. Thus, the function $F(a)$ initially set to 2 for the inclusion of $\boldsymbol{p}$ and $\boldsymbol{q}$ as inliers will be automatically increased by 1 because of the inclusion of $\boldsymbol{r}$.

- Suppose that $\boldsymbol{p}, \boldsymbol{q}$ and $\boldsymbol{r}$ are not colinear, but there is no intersection between $L_{p q}^{0}$ and $L_{r}$ in $Q_{p q} ; L_{r}$ is parallel to $L_{p q}^{0}$. We treat it in the same manner as the first degenerate case of digital line fitting; if $L_{\boldsymbol{r}}$ is between $L_{\boldsymbol{p} \boldsymbol{q}}^{0}$ and $L_{\boldsymbol{p} \boldsymbol{q}}^{1}$ (resp. $L_{p q}^{2}$ ), then we set the initial value of $F(a)$ to 3 when $l=1$ (resp. $l=2$ ); otherwise, we set it to 2 .

- When many lines $L_{r}$ enter or leave a strip at the same moment $a$, we apply the same procedure as the second degenerate case of digital line fitting.

Those modifications affect neither the time nor space complexity of the algorithm.

\section{Feasible digital line and plane parameters}

Once we obtained an optimal consensus set $\boldsymbol{C}$ for digital line or plane fitting to a given point set $\boldsymbol{S}$, we need the parameters of digital lines and planes fitted to $\boldsymbol{C}$ for many applications. In general, the continuous line and plane model such as (1) and (2) are used for estimating them, for example, by applying the least squared method (Hartley and Zisserman, 2003) to $\boldsymbol{C}$. However, we must be careful because this may change inliers. In such a case, a new $\boldsymbol{C}$ should be recalculated from a new estimated line or plane, so that the iterative procedure may be necessary for renewing $\boldsymbol{C}$ with consecutive re-estimated line or plane parameters.

In our case, however, since we use the digital model such as (3) and (4) instead of (1) and (2), we do not need such an estimation procedure, and we need not to worry that parameter values obtained by $\boldsymbol{C}$ may produce a different $\boldsymbol{C}$. We can obtain all feasible solutions for the parameters of digital lines (resp. planes) fitted to an obtained optimal $\boldsymbol{C}$. By simply looking for all feasible solutions $(a, b)(\operatorname{resp} .(a, b, c))$, that satisfy the inequalities of (3) (resp. (4)) for all $(x, y) \in \boldsymbol{C}$ (resp. $(x, y, z) \in \boldsymbol{C})$.

Such feasible solutions of digital lines and planes are called preimages. It is known that preimages of digital lines have interesting properties. For instance, a preimage of a digital line forms a convex polygon in the dual space that has at most four vertices (Dorst and Smeulders, 1984). However for digital planes, the structure of their preimages are more complex than that of digital lines (Gerard et al., 2008); we even do not know the maximum number of vertices or facets of a convex polyhedron that constitutes a preimage of a digital plane.

\section{Experiments}

This section presents the $2 \mathrm{D}$ and $3 \mathrm{D}$ experiments. We cannot avoid using colors in the figures shown in this section, which can be seen correctly at the on-line version of the paper.

\section{A. 2D noisy image of digitized lines}

We first tested our method with an image of size $102 \times 102$ originally made from two digital lines defined by a set of points $(x, y) \in Z^{2}$ satisfying either $0 \leq \frac{1}{2} x+y+50 \leq w$ or $0 \leq-\frac{4}{5} x+y+50 \leq w$, $w=0.999$. We then randomly added and removed 2000 points as noise for the image, and finally obtained 1800 points. Our method is applied to fit a digital line to these points. The optimal consensus set is found using our method, as shown in Figure 15; it has 87 inliers. 
From those 87 inliers, we also calculated a set of feasible parameters of fitted digital lines; it is given as the convex polygon in the parameter space $(a, b)$ of (3) whose vertices are $\left(-\frac{1}{99},-\frac{4751}{99}\right),(0,-49),(0,-48)$, $\left(\frac{1}{99},-\frac{4852}{99}\right)$.

We compared our result with that of RANSAC. For comparison, the tolerance of RANSAC is set to 0.5 ; this value specifies the maximum distance of inliers from a fitted line. In this experiment, we use the continuous line model of $a x+b y+c=0$ as in conventional RANSAC methods, and the vertical or horizontal distance as well as our method. Figure 16 shows the RANSAC results after 37587 iterations. The number of inliers is 34 and the parameters of the line are: $\quad a=-0.131175, b=0.149704, c=$ -0.989991 . It should be noted that with our method all the feasibility parameters can be exactly computed from the inliers, while RANSAC finds only one parameter set. Moreover, the 34 inliers obtained by RANSAC are far from the optimal result of our digital line. In fact, this is due to the fact that RANSAC is based on a random sampling, which provides no guarantee of optimality. However, the computation time is relatively rapid, thanks to its probabilistic strategy. Thus, in cases where there is a lot of noise, RANSAC should be avoided, whereas the use of RANSAC may be justified when it is sufficient to obtain an approximate solution for practical reasons,

\section{B. 2D real image}

We then tested our method with respect to a real image, as shown in Figure 17 (left), whose size is $520 \times 693$. Before applying our method, edge detection and mathematical morphological filtering are done for this image; the number of points in the image after this preprocessing is 5572 points. Our method is then applied in order to fit a digital line to the set of points. Figure 17 (right) shows the optimal consensus set, which includes 602 inliers, for digital line fitting. The distance $w$ was set to 1 .

\section{2D Polygonal contour images}

We also tested polygonalisation using our method. It is tested using an iterative procedure by applying our method; after each iteration, we take the inliers off and apply our method to the remaining points. Figure 18 (left) shows the original polygonal contour image containing some noise whose size is $497 \times 456$. Figure 18 (right) shows the result after six iterations of applying our method for the polygonalisation. The consensus set obtained after each iteration is colored in red, blue, yellow, pink, cyan and green, respectively. The number of all points is 1960 , and the sizes of the consensus sets are 297, 264, 186, 180, 119, and 104, respectively. The distance $w$ is set to be 1 .

\section{3D real images}

For the 3D experiments, we applied our proposed method to two example data, such as a 3D discrete point cloud and a 3D binary digital image.

The first example is a 3D discrete point cloud in Figure 19, which is obtained after a planar surface segmentation of a range image of blocks (Kenmochi et al., 2008). The number of points in the cloud is 12859 , and they are segmented into thirteen planar surfaces, which are illustrated in Figure 19 with points in different colors, except for those colored in light green that are detected as edge points. For each of these thirteen sets, we fitted a digital plane. We see the corresponding planes in Figure 20, and the number of points for each segmented surface and the size of its optimal consensus set in Table 1. In Figure 21, we also see that the fitted plane for the blue segmented surface points in Figure 19: inliers are colored blue while outliers are in pink.

We also applied our method to a 3D image extracted from a polymer foam observed in X-ray microtomography, on which homotopic thinning and surface decomposition were applied (Plougonven et al. (2006)). Figure 22 shows a cross section of the original image and Figure 23 shows a 3D binary image obtained after homotopic thinning and surface decomposition; the image is cut into two parts for visualization. Among around 400 sets of points forming surfaces in the entire image, we choose a part, as illustrated in Figure 24, including 17 decomposed surfaces for digital plane fitting. We show the fitted planes in Figure 25, and the number of points and the optimal consensus set size for each segmented surface in Table 2. For both the examples, we set $w=1$.

\section{Conclusions}


In this paper we have exposed a new method for line and plane fitting on discrete data such as bitmap images using a digital geometry (DG) approach. The DG approach allows practitioners to separate effects due to digitization on the one hand and noise on the other. Using our approach, we have proposed an optimal fitting method from the point of view of the maximal consensus set: we are guaranteed to fit a digital line or plane with the least amount of outliers. The 3D algorithm is based on the same idea as the 2D algorithm however some extensions are done to adapt the algorithm to a $2 \mathrm{D}$ dual problem and to cope with the different degenerate cases. The 2D and 3D algorithms has a complexity that are identical to parameter-less traditional plane-fitting algorithms such as least median of squares regression (Rousseeuw, 1984), but allows us to define a digital line or plane exactly, in the presence of outliers. Future work will include improving algorithmic complexities and more complete applications such as optimal polygonalization or polyhedrization by choosing a good value for $w$ automatically, and image registration considering all feasible digital line and plane parameters.

\section{ACKNOWLEDGEMENTS}

This work was accomplished while the first author was a post-doc at Université Paris-Est, Laboratoire d'Informatique Gaspard-Monge, ESIEE-CNRS, Paris. A preliminary version of this paper was presented at the 13th International workshop on Combinatorial Image Analysis (Zrour et al., 2009).

The authors express their thanks to Mr. John Chaussard, who supplied the 3D image of decomposed surfaces extracted from a polymer foam used in the experiments. A part of this work has been supported by the french National Research Agency projects, ANR05-BLAN-0071(SURF) and BLAN07-2-184378 (MICROFISS).

\section{REFERENCES}

P. Bhowmick and P. Bhaattacharya, Fast polygonal approximation of digital curves using relaxed straightness properties, IEEE Transactions on Pattern Analysis and Machine Intelligence, 29, 9 (2007), 1590-1602.
S. Boyd and L. Vandenberghe, Convex optimization, Cambridge University Press (2004).

L. Buzer, A linear incremental algorithm for naive and standard digital lines and planes recognition, Graphical Models, 65 (2003), 61-76.

L. Buzer, A simple algorithm for digital line recognition in the general case, Pattern Recognition, 40 (2006), 1675-1684.

M. De Berg, O. Cheong, M. Van Kreveld and M. Overmars, Computational Geometry: Algorithms and Applications, Springer-Berlag, Berlin Heidelberg (2008).

I. Debled Rennesson, F. Feschet and J. Rouyer-Degli, Optimal blurred segments decomposition of noisy shapes in linear time, Computer \& Graphics, 30 (2006), $30-36$.

L. Dorst and A.W.M. Smeulders, Discrete Representation of straight lines, IEEE Transactions on Pattern Analysis and Machine Intelligence, 6, 4 (1984), 450-463.

M.A. Fischler and R.C. Bolles, Random sample consensus: a paradigm for model fitting with applications to image analysis and automated cartography, Communications of the ACM, 24, 6 (1981), 381-395.

Y. Gerard, I. Debled Rennesson and P. Zimmermann, An elementary digital plane recognition algorithm, Discrete Applied Mathematics, 151, Issue 1-3 (2005), 169-183.

Y. Gerard, F. Feschet and D. Coeurjolly, GiftWrapping Based Preimage Computation Algorithm, Proceedings of International Conference on Discrete Geometry for Computer Imagery, Springer-Verlag, LNCS 4992 (2008), 310-321.

R. Hartley and R. Zisserman, Multiple view geometry in computer vision, Cambridge University Press (2003). 
Y. Kenmochi, L. Buzer, A. Sugimoto and I. Shimizu, Discrete plane segmentation and estimation from a point cloud using local geometric patterns. International Journal of Automation and Computing, 5, 3 (2008), 246-256.

R. Klette and A. Rosenfeld, Digital Geometry: Geometric Methods for Digital Picture Analysis, Morgan Kauffmann, San Francisco (2004).

K. Köster and M. Spann, An approach to Robust Clustering - Application to Range Image Segmentation, IEEE Transactions on Pattern Analysis and Machine Intelligence. 22, 5, (2000), 430-444.

E. Plougonven, D. Bernard and P. Viot, Quantitative analysis of the deformation of polypropylene foam under dynamic loading, Progress in biomedical optics and imaging, 7, 38 (2006).

W.H. Press, S.A. Teukolsky, W.T. Vetterling and B.P. Flannery, Numerical recipes: the art of scientific computing, third edition, Cambridge University Press (2007).

L. Provot, L. Buzer, I. Debled Rennesson, Recognition of blurred pieces of discrete planes, 13th International Conference on Discrete Geometry for Computer Imagery, 4245 (2006), 65-76.

J.P. Reveilles, Géométrie discrète, calcul en nombres entiers et algorithmique, Thèse d'Etat, Université Louis Pasteur, Strasbourg, France (1991).

P.J. Rousseeuw, Least median of squares regression, Journal of the American statistical association, 79, 388 (1984), 871-880.

H.Y. Shum, K. Ikeuchi and R. Reddy, Principal component analysis with missing data and its application to polyhedral object modeling, IEEE Transactions on Pattern Analysis and Machine Intelligence, 17, 9 (1995), 854-867.
I. Sivignon, F. Dupont and J.M. Chassery, Decomposition of a three-dimensional discrete object surface into discrete plane pieces, Algorithmica, 38 (2004), 25-43.

D.L. Souvaine and J.M. Steele, Time- and spaceefficient algorithms for least median of squares regression, Journal of the American Statistical Association, 82, 399 (1987), 794-801.

B. Zitova and J. Flusser, Image registration methods: a survey, Image and Vision Computing 21 (2003), 9771000 .

R. Zrour, Y. Kenmochi, H. Talbot, L. Buzer, Y. Hamam, A. Sugimoto and I. Shimizu, Optimal consensus set for digital line fitting, Progress in Combinatorial Image Analysis, P. Wiederhold and R.P. Barneva (Editors), Research Publishing, Singapore, (2009), 93-105.

R. Zrour, Y. Kenmochi, H. Talbot, L. Buzer, Y. Hamam, A. Sugimoto and I. Shimizu, Optimal consensus set for digital plane fitting, proceedings of 3DIM'09 ICCV Satallite workshop (2009), 1817-1824. 


\section{Figures captions}

Fig. 1. A digital line that has one critical point $\boldsymbol{p}_{1}$ and its rotated digital line with a second critical point $\boldsymbol{p}_{2}$.

Fig. 2. A digital line that has no critical point and its translated digital line with one critical point $\boldsymbol{p}_{\mathbf{1}}$.

Fig. 3. A digital plane with two critical points $\boldsymbol{p}_{1}$ and $\boldsymbol{p}_{2}$ on one of its support planes and its rotated digital plane that also has a third critical point $\boldsymbol{p}_{3}$.

Fig. 4. A digital plane with two critical points $\boldsymbol{p}_{1}$ and $\boldsymbol{p}_{2}$ on distinct support planes and its rotated digital plane that also a the third critical point $\boldsymbol{p}_{3}$.

Fig. 5. A digital plane with one critical point $\boldsymbol{p}_{1}$ and its rotated digital plane that also has a second critical point $\boldsymbol{p}_{2}$.

Fig. 6. A digital plane with no critical point and its translated digital plane that has one critical point $\boldsymbol{p}_{\mathbf{1}}$.

Fig. 7. A digital line of width $w$ in the primal space (left) corresponds to a vertical line segment of length $w$ in the dual space (right).

Fig 8. Digital lines on which a point $\boldsymbol{p}$ is a critical point in the primal space (left), and those corresponding vertical line segments of length $w$ in the dual space (right). In the dual space, a set of all such digital lines forms two strips, each of which is bounded by two lines $L_{\boldsymbol{p}}^{0}$ and $L_{\boldsymbol{p}}^{i}$ for $i=1,2$.

Fig. 9. Three points $\boldsymbol{p}, \boldsymbol{q}, \boldsymbol{r}$ in the primal space (left), and the corresponding lines $L_{\boldsymbol{p}}^{0}, L_{\boldsymbol{q}}^{0}$ and $L_{\boldsymbol{r}}^{0}$ in the dual space, with their intersections $\boldsymbol{\sigma}_{\boldsymbol{q}}^{i}, \boldsymbol{\sigma}_{\boldsymbol{r}}^{i}$ for $i=0,1$ (right).

Fig. 10. Algorithm 1: digital line fitting.

Fig. 11. A digital plane in the primal space (left) corresponds to a vertical line segment of length $w$ in the dual space (right). 
Fig. 12. All the digital planes with two critical points $\boldsymbol{p}$ and $\boldsymbol{q}$ in the primal space (left) correspond to a set of vertical line segments of length w having one of its endpoints on the intersection line of the two planes $P_{\boldsymbol{p}}$ and $P_{\boldsymbol{q}}$ (right).

Fig. 13. Four points $\boldsymbol{p}, \boldsymbol{q}, \boldsymbol{r}$ and $\boldsymbol{s}$ in the primal space (left), and their interpretations in the cross-section $Q_{p \boldsymbol{q}}$ of the dual space (right). $Q_{\boldsymbol{p}}$ is made as the plane that contains the intersection line $L_{\boldsymbol{p} q}^{0}$ of $P_{\boldsymbol{p}}$ and $P_{\boldsymbol{q}}$ and the parallel direction to the c-axis, as illustrated in Figure 12. In $Q_{\boldsymbol{p} \boldsymbol{q}}$, all the digital planes having $\boldsymbol{p}$, and $\boldsymbol{q}$ as critical points are represented by the strips each of which is bounded by $L_{\boldsymbol{p} \boldsymbol{q}}^{0}$ and either of its parallel lines $L_{\boldsymbol{p} \boldsymbol{q}}^{1}$ and $L_{\boldsymbol{p} \boldsymbol{q}}^{2}$. The other points $r$ and $s$ in the primal space are represented by the two lines $L_{r}$ and $L_{\boldsymbol{s}}$ in $Q_{\boldsymbol{p q}}$.

Fig. 14. Algorithm 2 : digital plane fitting.

Fig. 15. The optimal consensus set (87 red points) obtained by our method for digital line fitting to a noisy image of digitized lines containing 1800 points.

Fig. 16. A consensus set (34 red points) obtained by RANSAC for line fitting after 37587 iterations to the same image of Figure 15.

Fig. 17. An original image (left), and its optimal consensus set, in red color, of digital line fitting (right).

Fig. 18. A polygonal contour image with noise (left), and its result after six iterations of applying our method: the optimal consensus set obtained after each iteration is in red, blue, yellow, pink, cyan and green, respectively (right).

Fig. 19. Planar surface segmentation of a 3D discrete point cloud: the number of points is 12859 , and they are segmented into thirteen planar surfaces whose points are in different colors, except for those colored in light green that are detected as edge points. 
Fig. 20. Fitted planes of segmented planar surface in Figure 19.

Fig. 21. The fitted plane with its optimal consensus set for the blue segmented surface points in Figure 20: inliers are colored blue while outliers are colored pink.

Fig. 22. A cross section of a 3D image extracted from a polymer foam observed in X-ray micro-tomography.

Fig. 23. The 3D binary image obtained after homotopic thinning and surface decomposition applied on the image in Figure 22: the image is cut into two parts for visualization.

Fig. 24. Selected decomposed surfaces, which is a part of the 3D binary image in Figure 23, for digital plane fitting.

Fig. 25. Fitted digital planes for decomposed surfaces shown in Figure 24.

Table 1. The number of points for each segmented surface in Figure 20 and the size of its optimal consensus set.

Table 2. The number of points and the optimal consensus set size for each decomposed surface in Figure 25. 


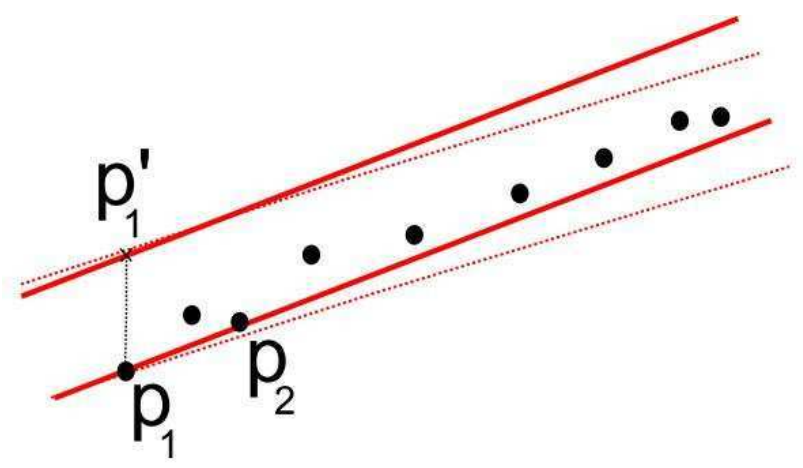

Fig. 1. A digital line that has one critical point $\boldsymbol{p}_{\mathbf{1}}$ and its rotated digital line with a second critical point $\boldsymbol{p}_{\mathbf{2}}$.

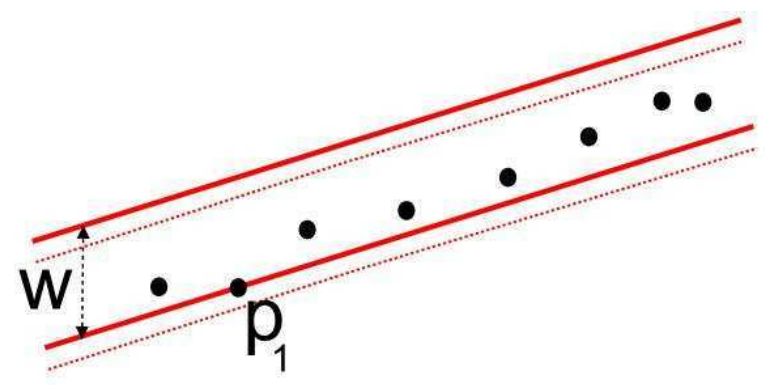

Fig. 2. A digital line that has no critical point and its translated digital line with one critical point $\boldsymbol{p}_{\mathbf{1}}$.

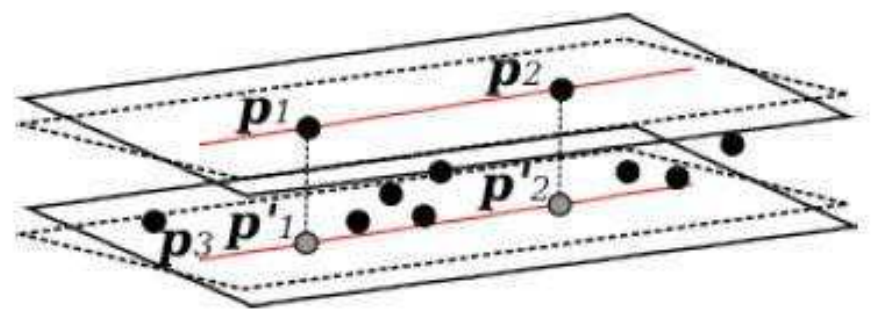

Fig. 3. A digital plane with two critical points $\boldsymbol{p}_{1}$ and $\boldsymbol{p}_{2}$ on one of its support planes and its rotated digital plane that also has a third critical point $\boldsymbol{p}_{\mathbf{3}}$.

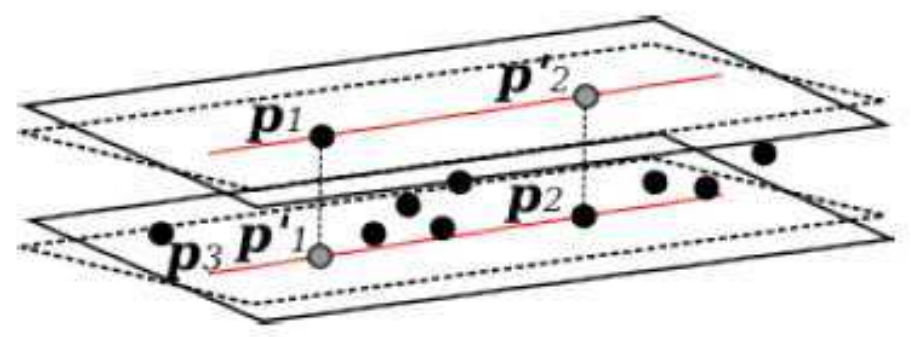


Fig. 4. A digital plane with two critical points $\boldsymbol{p}_{1}$ and $\boldsymbol{p}_{2}$ on distinct support planes and its rotated digital plane that also a the third critical point $\boldsymbol{p}_{3}$.

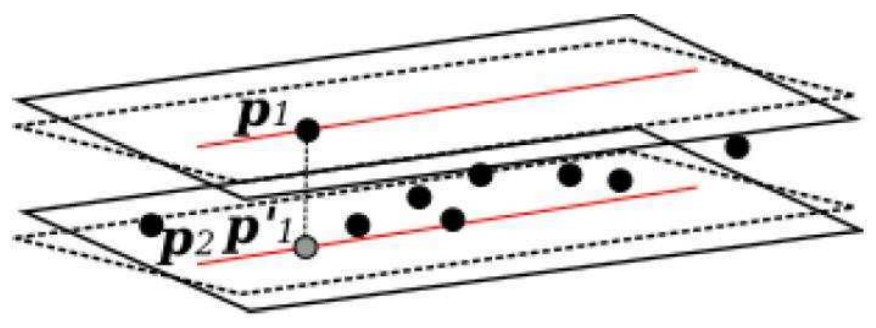

Fig. 5. A digital plane with one critical point $\boldsymbol{p}_{1}$ and its rotated digital plane that also has a second critical point $\boldsymbol{p}_{2}$.

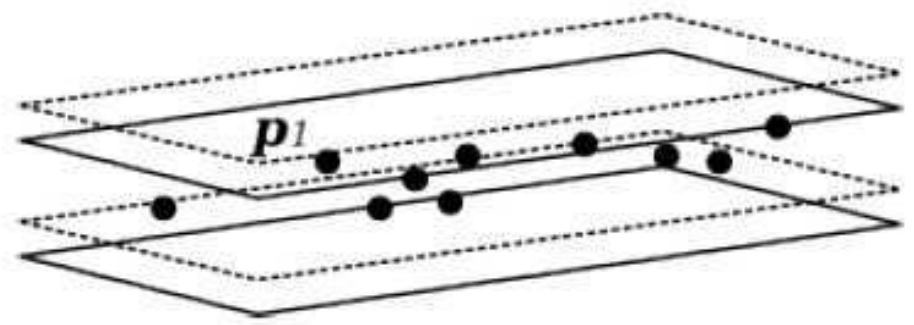

Fig. 6. A digital plane with no critical point and its translated digital plane that has one critical point $\boldsymbol{p}_{\mathbf{1}}$.
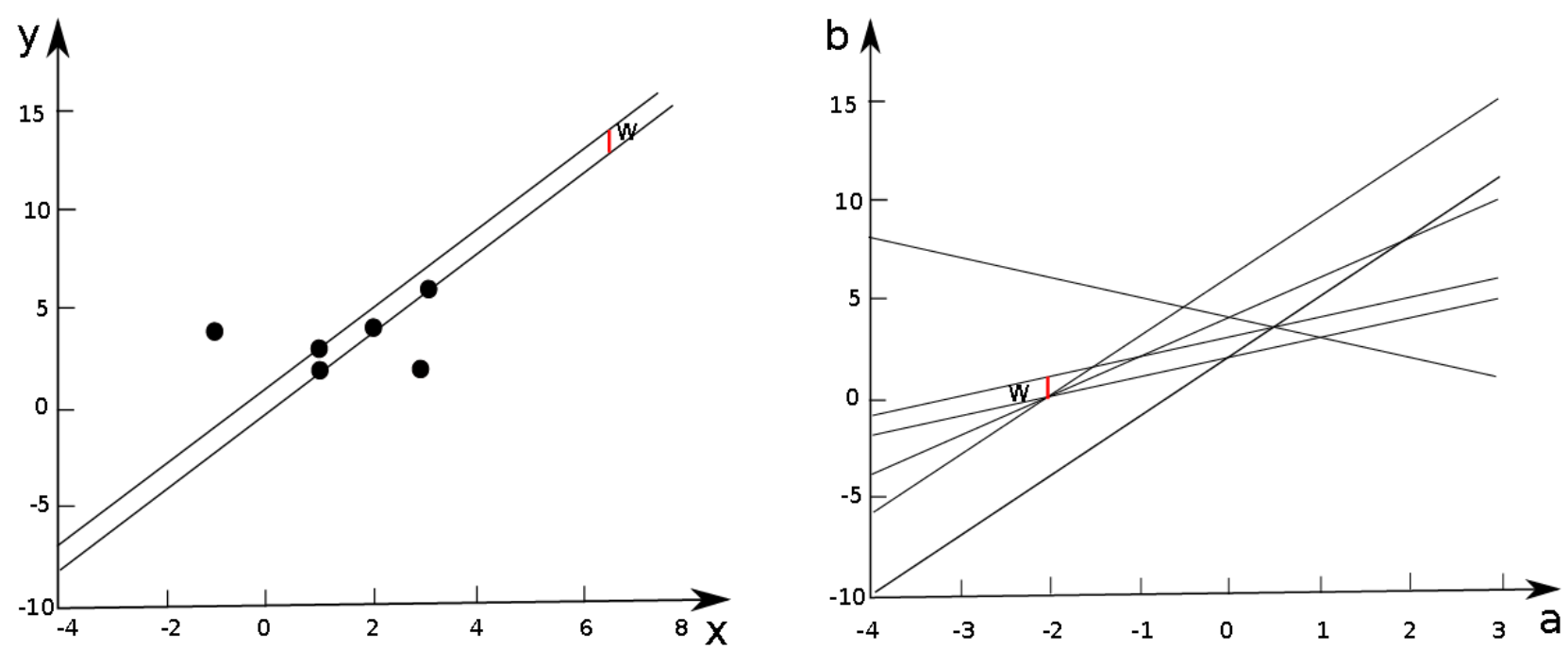

Fig. 7. A digital line of width $w$ in the primal space (left) corresponds to a vertical line segment of length $w$ in the dual space (right). 

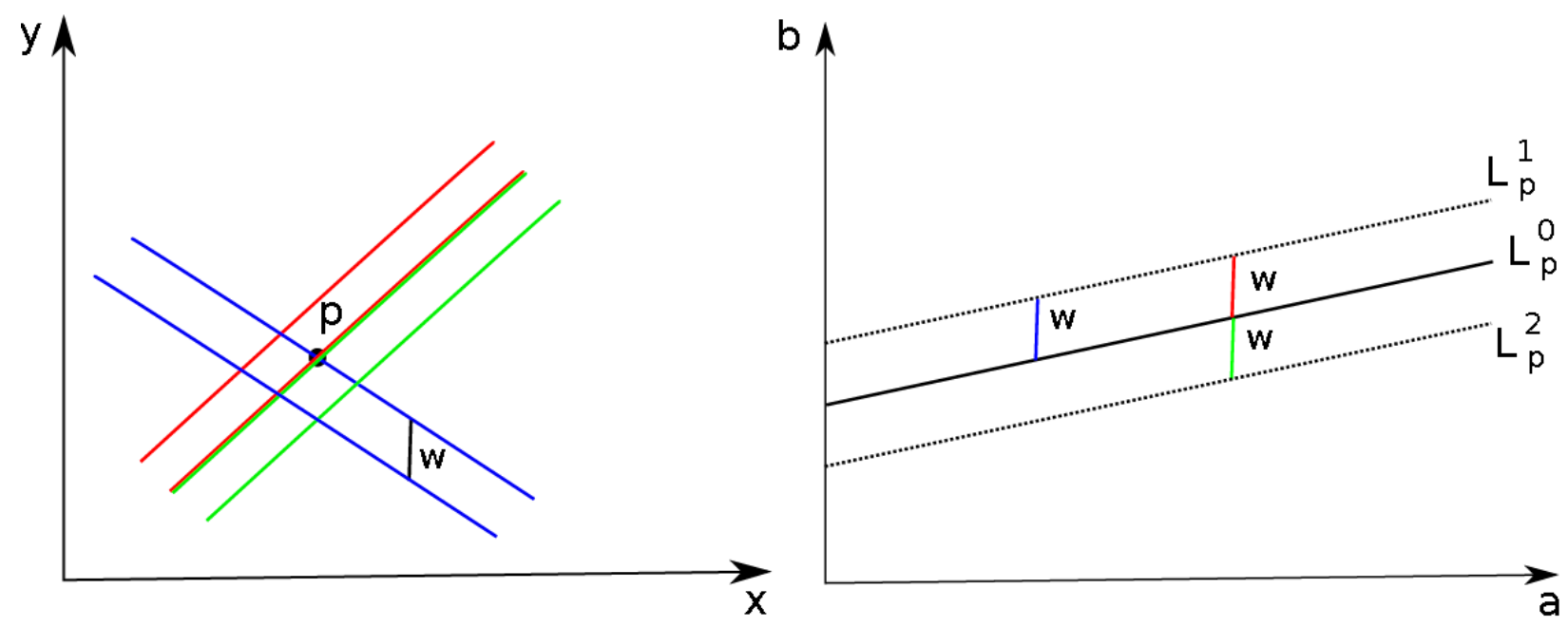

Fig 8. Digital lines on which a point $\boldsymbol{p}$ is a critical point in the primal space (left), and those corresponding vertical line segments of length $w$ in the dual space (right). In the dual space, a set of all such digital lines forms two strips, each of which is bounded by two lines $L_{p}^{0}$ and $L_{p}^{i}$ for $i=1,2$.
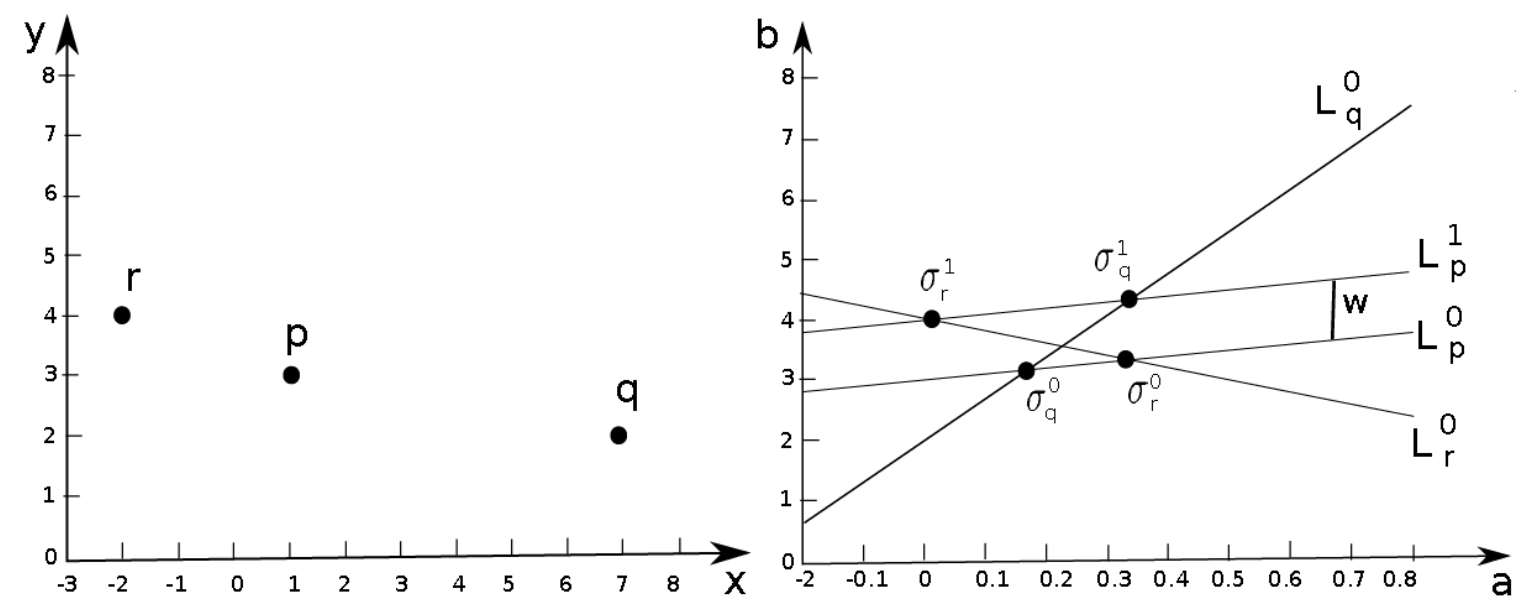

Fig. 9. Three points $\boldsymbol{p}, \boldsymbol{q}, \boldsymbol{r}$ in the primal space (left), and the corresponding lines $L_{\boldsymbol{p}}^{0}, L_{\boldsymbol{q}}^{0}$ and $L_{\boldsymbol{r}}^{0}$ in the dual space, with their intersections $\boldsymbol{\sigma}_{\boldsymbol{q}}^{i}, \boldsymbol{\sigma}_{\boldsymbol{r}}^{i}$ for $i=0,1$ (right). 


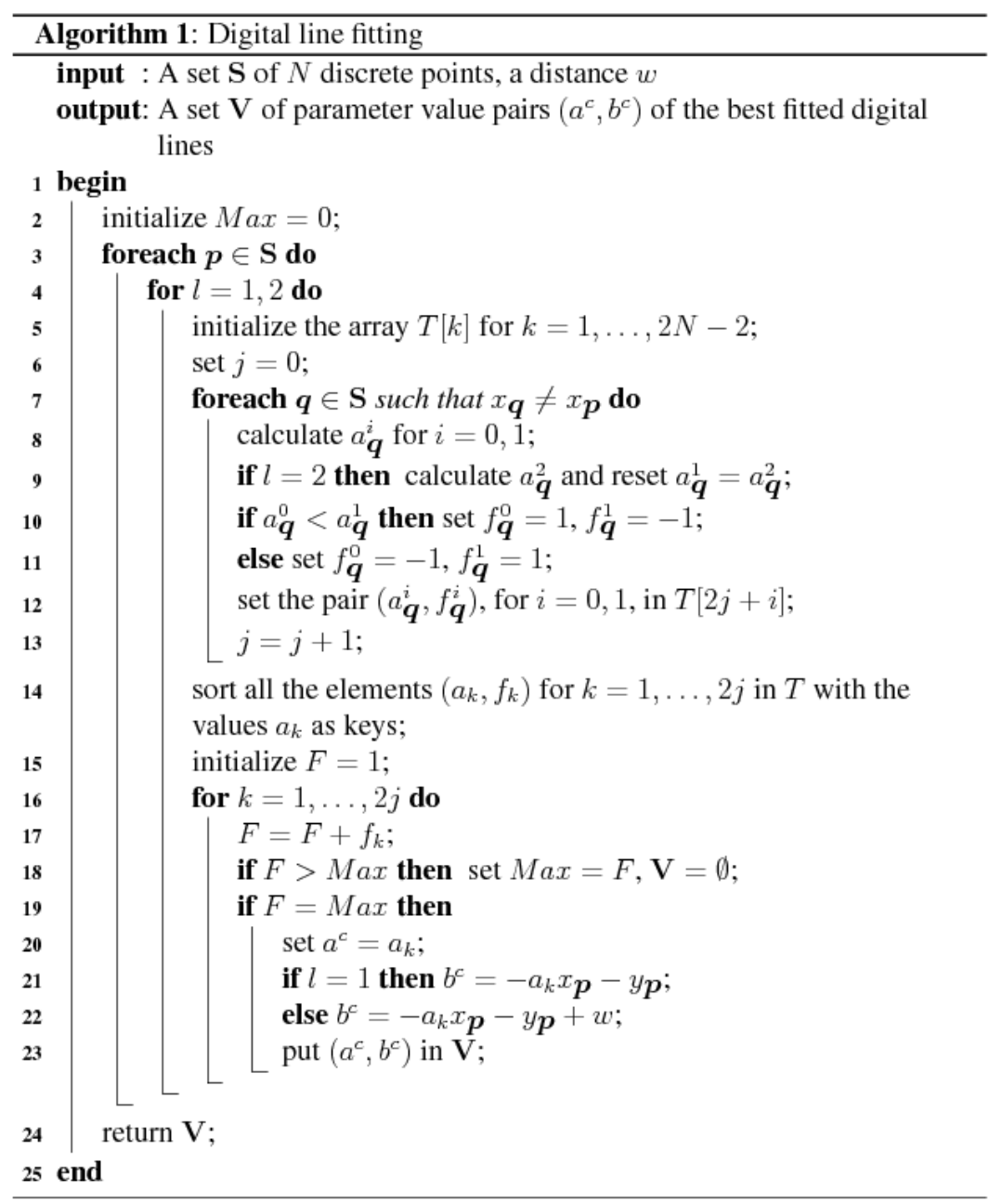

Fig. 10. Algorithm 1: digital line fitting.
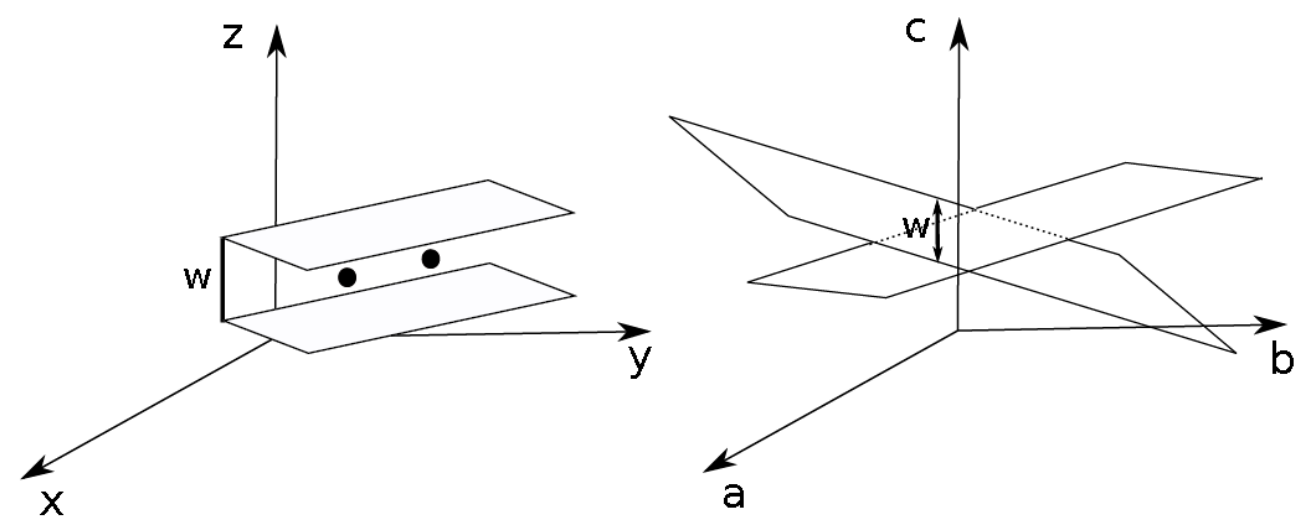

Fig. 11. A digital plane in the primal space (left) corresponds to a vertical line segment of length $w$ in the dual space (right). 

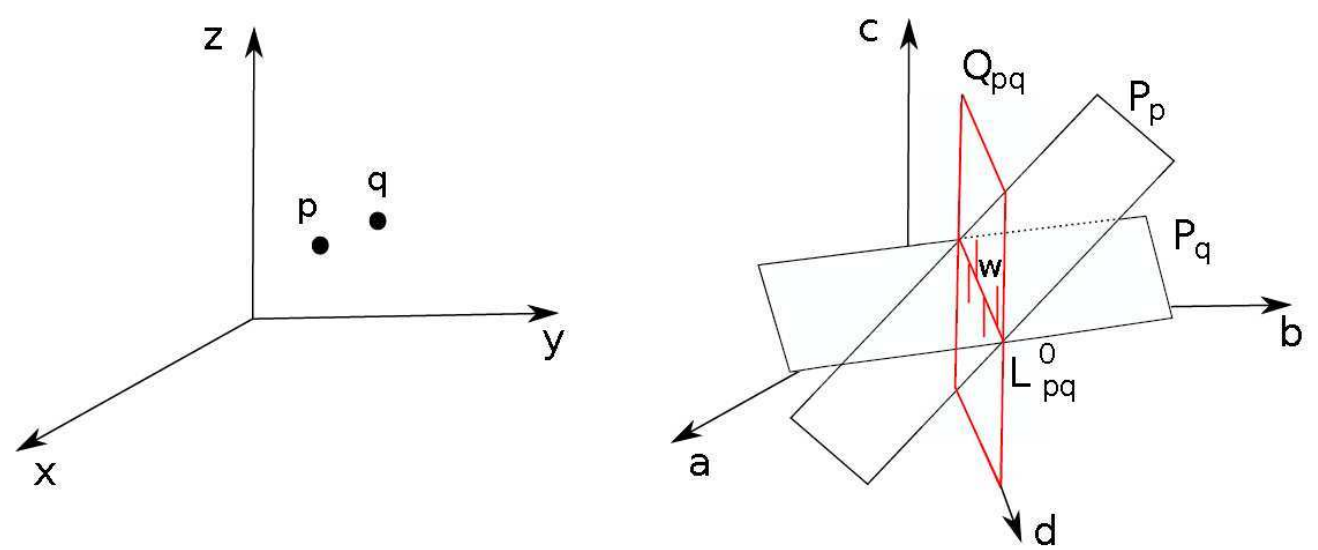

Fig. 12. All the digital planes with two critical points $\boldsymbol{p}$ and $\boldsymbol{q}$ in the primal space (left) correspond to a set of vertical line segments of length w having one of its endpoints on the intersection line of the two planes $P_{\boldsymbol{p}}$ and $P_{\boldsymbol{q}}$ (right).
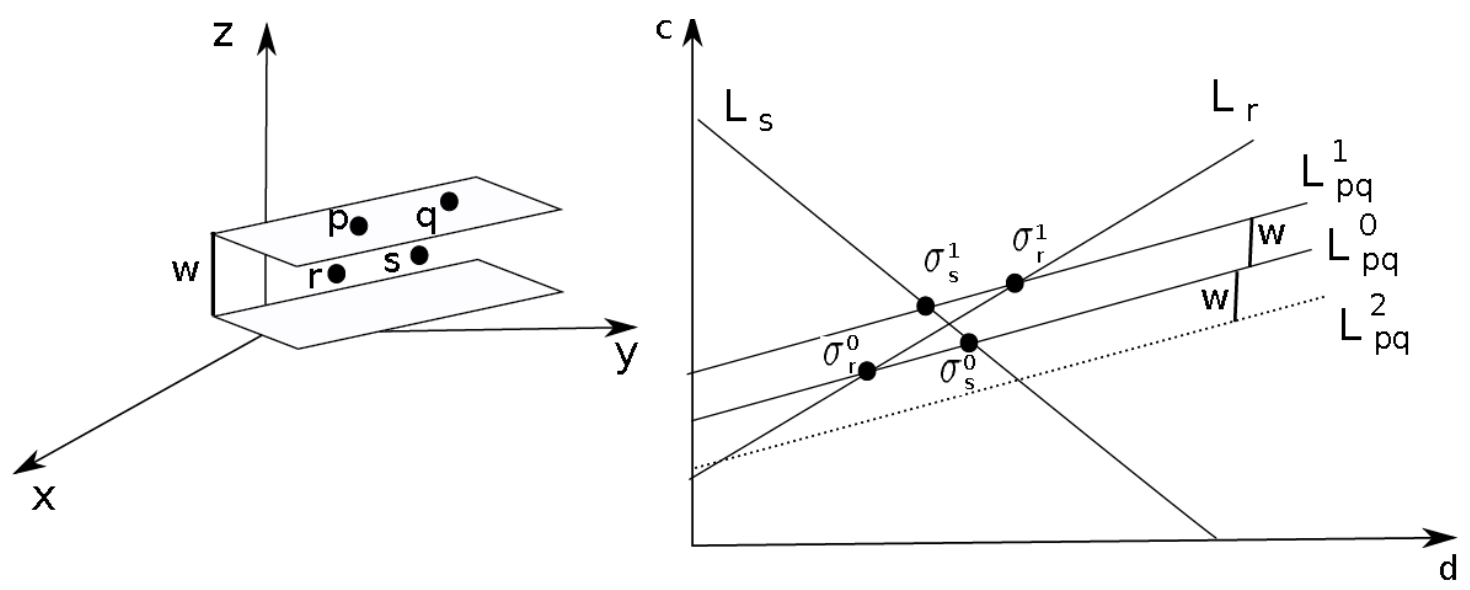

Fig. 13. Four points $\boldsymbol{p}, \boldsymbol{q}, \boldsymbol{r}$ and $\boldsymbol{s}$ in the primal space (left), and their interpretations in the cross-section $Q_{\boldsymbol{p} q}$ of the dual space (right). $Q_{\boldsymbol{p} \boldsymbol{q}}$ is made as the plane that contains the intersection line $L_{\boldsymbol{p} \boldsymbol{q}}^{0}$ of $P_{\boldsymbol{p}}$ and $P_{\boldsymbol{q}}$ and the parallel direction to the c-axis, as illustrated in Figure 12. In $Q_{\boldsymbol{p} \boldsymbol{q}}$, all the digital planes having $\boldsymbol{p}$, and $\boldsymbol{q}$ as critical points are represented by the strips each of which is bounded by $L_{p q}^{0}$ and either of its parallel lines $L_{p q}^{1}$ and $L_{p q}^{2}$. The other points $r$ and $s$ in the primal space are represented by the two lines $L_{\boldsymbol{r}}$ and $L_{\boldsymbol{s}}$ in $Q_{\boldsymbol{p q}}$. 


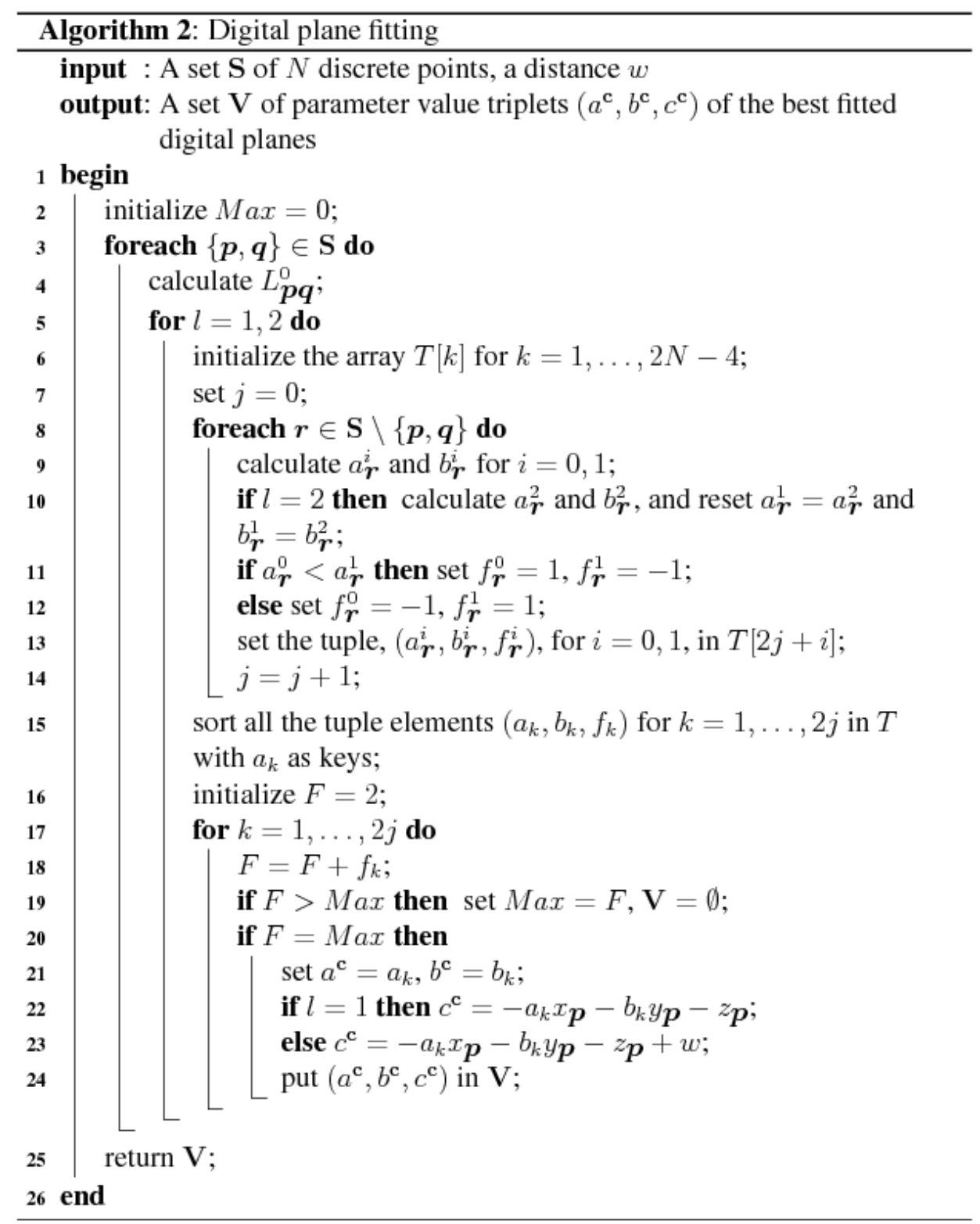

Fig. 14. Algorithm 2 : digital plane fitting. 


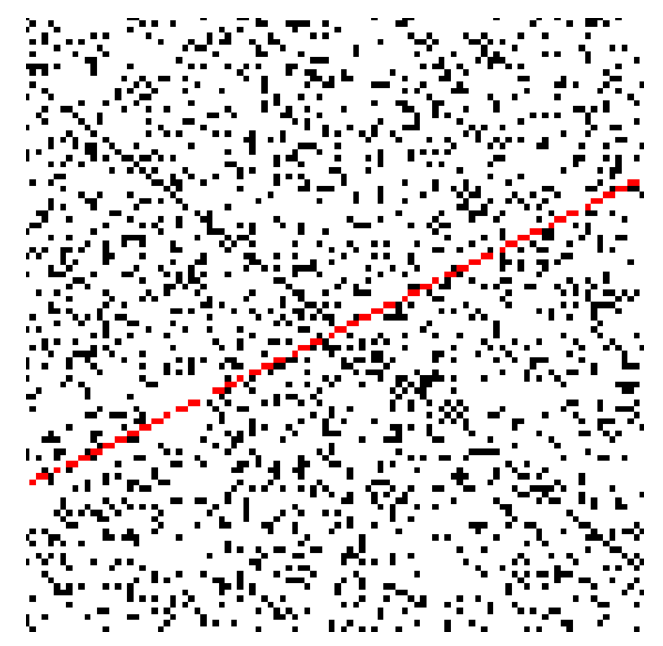

Fig. 15. The optimal consensus set (87 red points) obtained by our method for digital line fitting to a noisy image of digitized lines containing 1800 points.

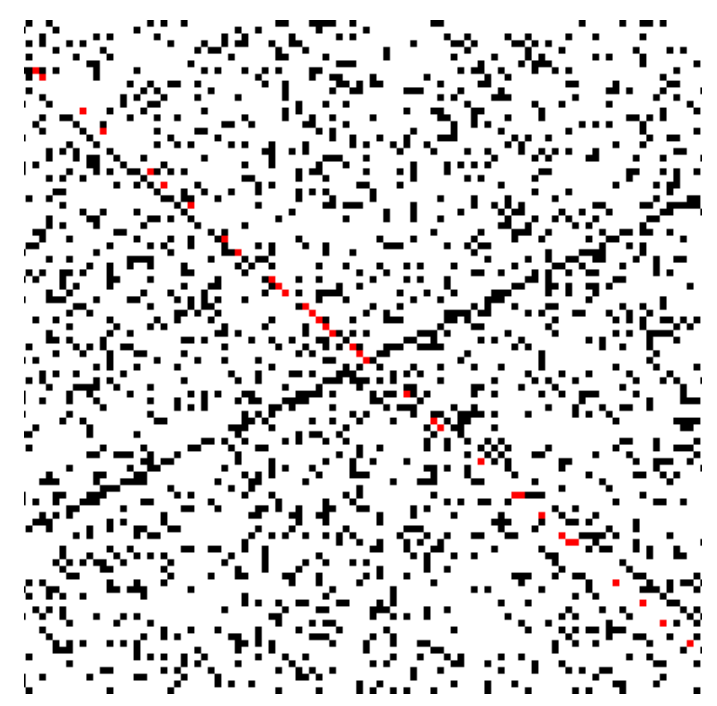

Fig. 16. A consensus set (34 red points) obtained by RANSAC for line fitting after 37587 iterations to the same image of Figure 15. 

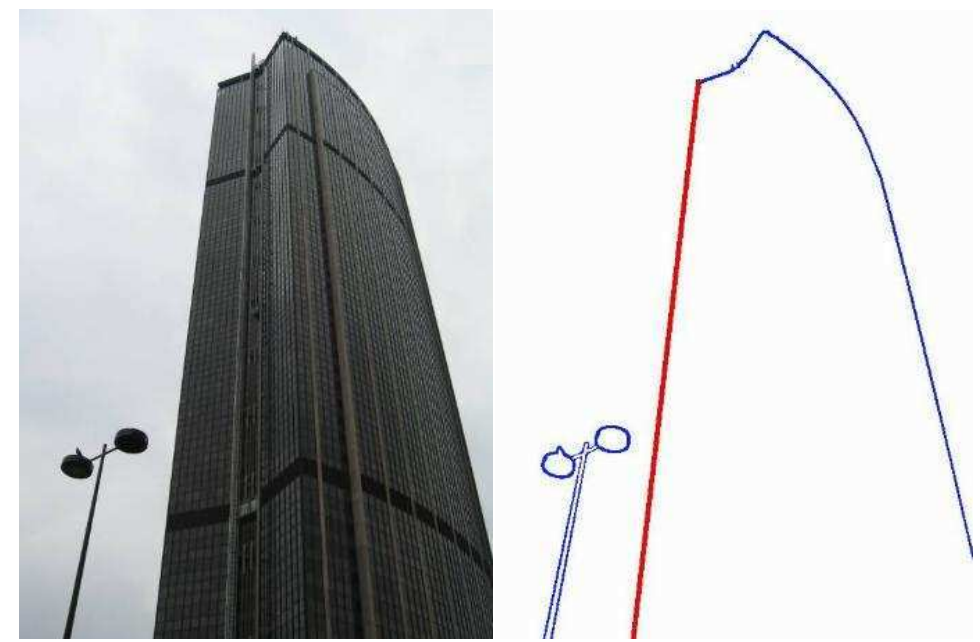

Fig. 17. An original image (left), and its optimal consensus set, in red color, of digital line fitting (right).
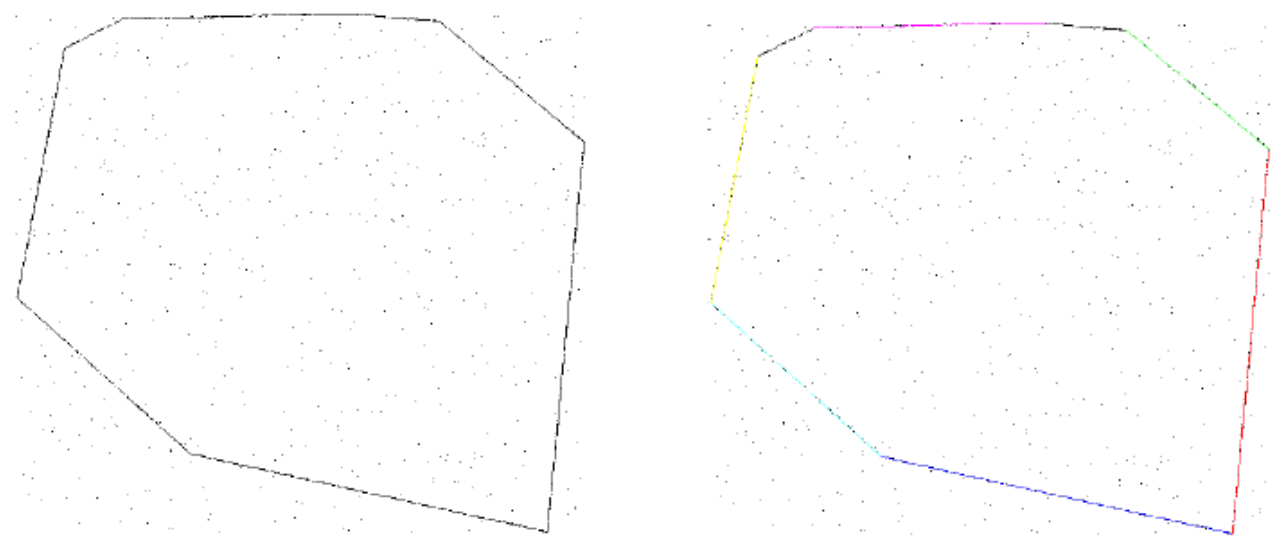

Fig. 18. A polygonal contour image with noise (left), and its result after six iterations of applying our method: the optimal consensus set obtained after each iteration is in red, blue, yellow, pink, cyan and green, respectively (right). 


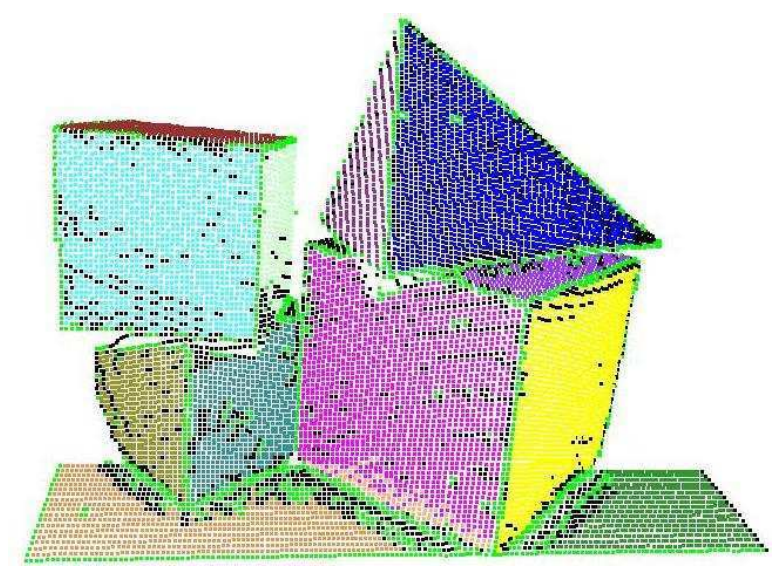

Fig. 19. Planar surface segmentation of a 3D discrete point cloud: the number of points is 12859 , and they are segmented into thirteen planar surfaces whose points are in different colors, except for those colored in light green that are detected as edge points.

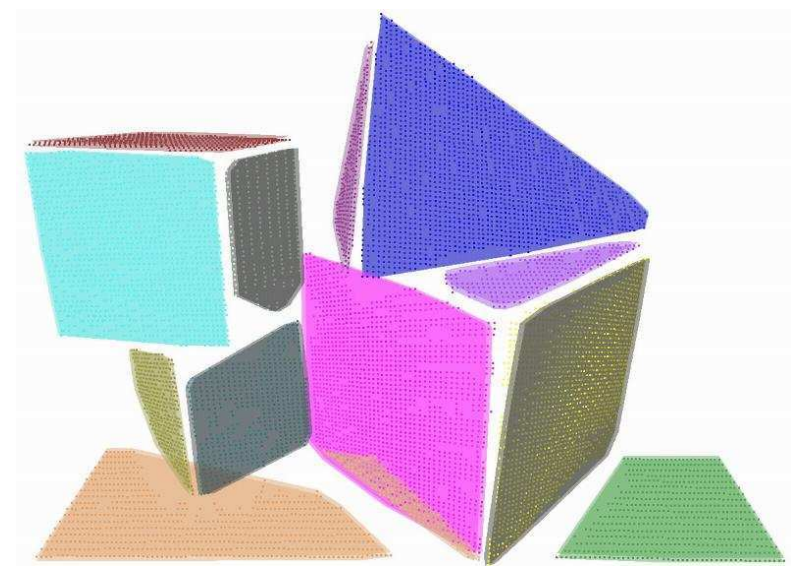

Fig. 20. Fitted planes of segmented planar surface in Figure 19.

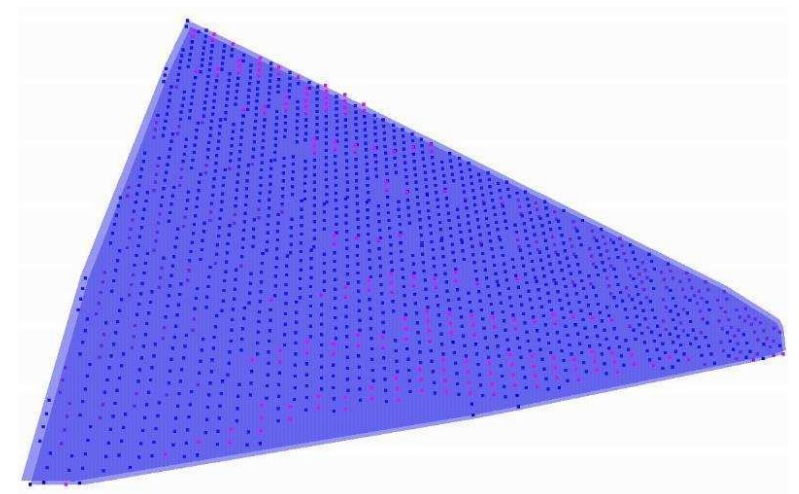

Fig. 21. The fitted plane with its optimal consensus set for the blue segmented surface points in Figure 20: inliers are colored blue while outliers are colored pink. 


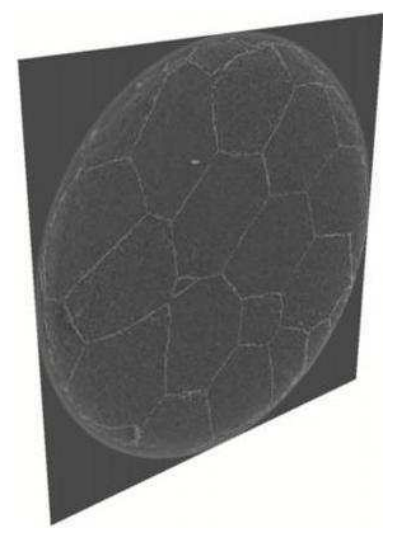

Fig. 22. A cross section of a $3 D$ image extracted from a polymer foam observed in X-ray micro-tomography.

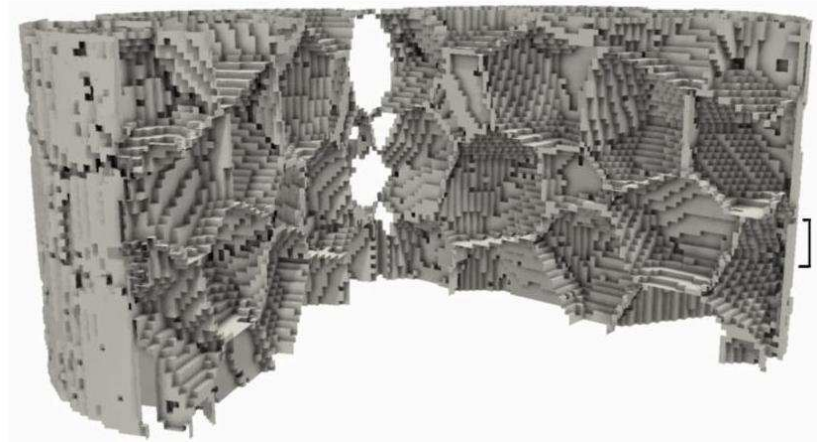

Fig. 23. The 3D binary image obtained after homotopic thinning and surface decomposition applied on the image in Figure 22: the image is cut into two parts for visualization.

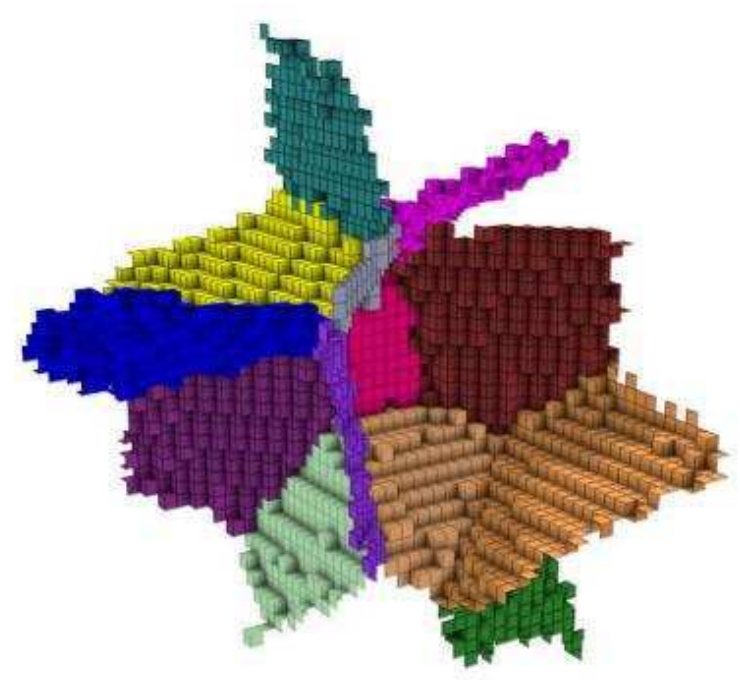

Fig. 24. Selected decomposed surfaces, which is a part of the 3D binary image in Figure 23, for digital plane fitting. 


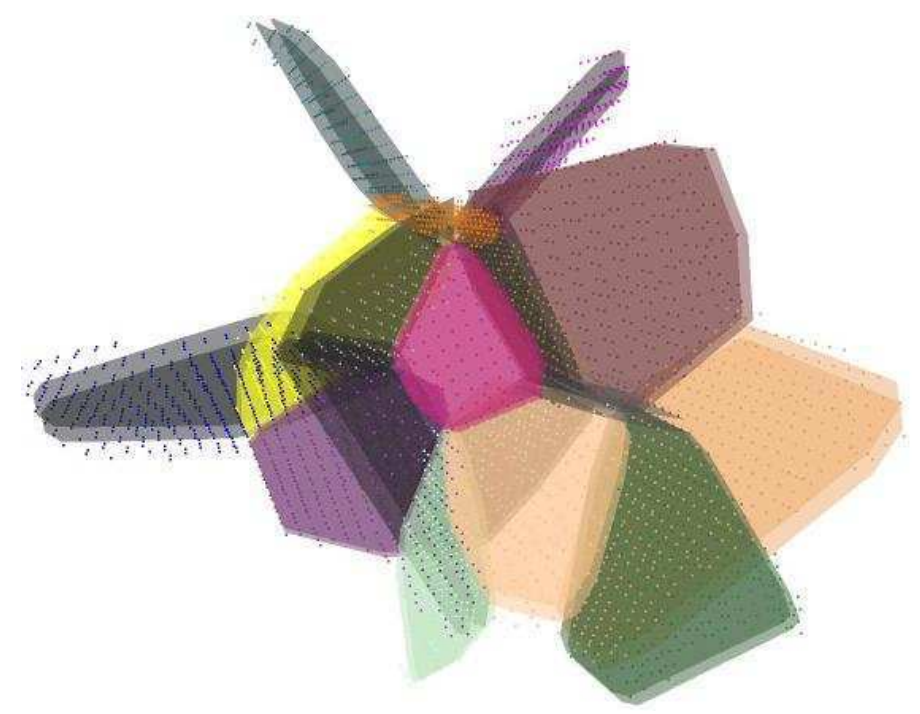

Fig. 25. Fitted digital planes for decomposed surfaces shown in Figure 24.

Table 1. The number of points for each segmented surface in Figure 20 and the size of its optimal consensus set.

\begin{tabular}{|c|c|c|}
\hline & Number of points & Opt. consensus set size \\
\hline Blue & 1770 & 1401 \\
\hline Yellow & 1578 & 1195 \\
\hline Pink & 1523 & 935 \\
\hline Pale blue & 1191 & 922 \\
\hline Orange & 699 & 693 \\
\hline Green & 573 & 573 \\
\hline Brown & 545 & 544 \\
\hline Turquoise & 536 & 512 \\
\hline Olive & 440 & 405 \\
\hline Purple & 248 & 245 \\
\hline Violet & 232 & 206 \\
\hline
\end{tabular}




\begin{tabular}{|c|c|c|}
\hline Moss green & 223 & 223 \\
\hline Cream & 101 & 97 \\
\hline
\end{tabular}

Table 2. The number of points and the optimal consensus set size for each decomposed surface in Figure 25.

\begin{tabular}{|c|c|}
\hline Number of points & Opt. consensus set size \\
\hline 541 & 269 \\
\hline 512 & 233 \\
\hline 439 & 208 \\
\hline 427 & 196 \\
\hline 427 & 200 \\
\hline 405 & 208 \\
\hline 377 & 159 \\
\hline 335 & 206 \\
\hline 333 & 169 \\
\hline 309 & 141 \\
\hline 308 & 168 \\
\hline 258 & 76 \\
\hline 220 & 104 \\
\hline 200 & 90 \\
\hline 198 & 61 \\
\hline 163 & 98 \\
\hline 104 & 71 \\
\hline
\end{tabular}

